Supplementary Material

\title{
Synthesis of Cyclopropanated [2.2.1] Heterobicycloalkenes: An Improved Procedure
}

\author{
Emily Carlson, ${ }^{\dagger}$ Guillaume Duret, ${ }^{\ddagger}$ Nicolas Blanchard, ${ }^{\ddagger}$ and William Tam ${ }^{*}{ }^{\dagger}$ \\ Guelph-Waterloo Centre for Graduate Work in Chemistry and Biochemistry, Department of \\ Chemistry, University of Guelph, Guelph, Ontario, Canada N1G 2W1 \\ Université de Strasbourg, Laboratoire de Chimie Moléculaire, CNRS UMR 7509, 25 rue \\ Becquerel, 67087 Strasbourg Cedex, France \\ ${ }^{\dagger}$ University of Guelph, Canada. \\ Université de Strasbourg, France.
}

\section{Contents}

General Information

S2

Full experimental detail and characterization data

S2-S11

${ }^{1} \mathrm{H},{ }^{2} \mathrm{H}$ and ${ }^{13} \mathrm{C}$ NMR spectra of cyclopropanes $\mathbf{2 d - j}$

S12-S19

Selective gradient NOE spectrum of cyclopropane $\mathbf{6 a}$

S20

${ }^{1} \mathrm{H}$ and ${ }^{13} \mathrm{C}$ NMR spectra of cyclopropanes 6a-c

S20-S24 
General Information: All reactions were carried out using a continuous flow apparatus under inert atmosphere. Commercial reagents and catalysts were used without further purification. Column chromatography was performed on $230-400$ mesh silica gel following standard flash column chromatography techniques, ${ }^{1}$ and analytical thin-layer chromatography (TLC) was performed on pre-coated silica gel $250 \mu \mathrm{m} 60 \mathrm{~F} 254$ aluminum plates, visualized by UV light and p-anisaldehyde stain. IR spectra were obtained as $\mathrm{NaCl}$ or $\mathrm{KBr}$ discs on a Nicolet 380-FTIR spectrometer, or as solids on an ALPHA platinum single reflection diamond ATR spectrophotometer. ${ }^{1} \mathrm{H},{ }^{2} \mathrm{H}$, and ${ }^{13} \mathrm{C}$ NMR spectra were recorded on Avance $400 \mathrm{MHz}$ or 600 $\mathrm{MHz}$ spectrometers equipped with cryoprobes and are reported in parts per million (ppm) from the solvent as internal standard $\left(\mathrm{CDCl}_{3}: \delta 7.24 \mathrm{ppm}\left({ }^{1} \mathrm{H}\right.\right.$ at $\left.400 / 600 \mathrm{MHz}\right)$ or $\delta 77.0 \mathrm{ppm}\left({ }^{13} \mathrm{C}\right.$ at 100/125 MHz)). HRMS samples were ionized by electron impact (EI) or electrospray ionization (ESI) as specified and detection of the ions was performed by time of flight (TOF).

General Procedure: HAZARD ALERT! Diazomethane can be fatal if inhaled and capable of detonation if appreciably concentrated. Refer to Figure S1: Reactor [C], equipped with a small stir bar, was charged with alkene $(1.2-17.7 \mathrm{mmol}), \mathrm{Pd}(\mathrm{OAc})_{2}(1 \mathrm{~mol} \%$ of alkene $)$ and tetrahydrofuran $(40 \mathrm{~mL})$ and was capped with septum [E]. To the outlet of reactor [C] was connected in series with Tygon tubing $[\mathrm{I}]$ an empty bubbler [J] to serve as a suck-back trap and a glass inlet tube $[\mathrm{K}]$ inserted into filter flask $[\mathrm{L}]$. Bubbler [L] was filled prior to setup with a glacial acetic acid-water mixture (1:1). The outlet of bubbler [L] was connected to a piece of Tygon tubing $\left[\mathrm{I}_{\mathrm{c}}\right]$ directed to the back of the fumehood. Reactor $[\mathrm{C}]$ was then securely fitted to the end of tube $\left[\mathrm{H}_{b}\right]$ while cooling its contents in an ice bath. Funnel [A] was filled with $25 \%$ $(12.5 \mathrm{M})$ aqueous sodium hydroxide (100-150 equivalents to alkene) ensuring that stopper [D]

\footnotetext{
${ }^{1}$ Still, W.C.; Kahn, M.; Mitra, A. J. Org. Chem. 1978, 43, 2923-2925.
} 
was tightly shut, and the funnel was capped with septum [E]. Flask [B], equipped with an extralarge stir bar, was charged with Diazald® (2.6-8 equivalents to alkene) and 95\% EtOH (50 mL), and the solution was stirred. Flask [B] was then fitted with a stopper [G] containing the inert gas inlet $[\mathrm{F}]$, tubing $\left[\mathrm{H}_{\mathrm{a}}\right]$, and addition funnel $[\mathrm{A}]$ assembly. The apparatus was securely clamped at both funnel $[\mathrm{A}]$ and flask [B] and a slow stream of argon was passed through the system such that $\sim 3$ bubbles per second (bps) were observed from tube $\left[\mathrm{H}_{\mathrm{a}}\right]$. Once a constant flow rate of 3-5 bps was established, 25\% sodium hydroxide solution was added from funnel [A] into flask [B] at a rate of 1-2 $\mathrm{mL} / \mathrm{min}$, maintaining efficient stirring and bubbling. Formation of the light yellow $\mathrm{CH}_{2} \mathrm{~N}_{2}$ gas was observed with the dissolution of Diazald®. Upon complete dissipation of any yellow colour (4-8 hours), the reaction was assessed by TLC for completion. Once the reaction was seen to be complete by TLC, both septa [E] were removed and the apparatus was left to vent any trace $\mathrm{CH}_{2} \mathrm{~N}_{2}$ (8-16 hours). Reactor [C] was removed and its contents were poured over Celite. The filter cake was washed with several portions $(4 \times 10-20 \mathrm{~mL})$ of $\mathrm{Et}_{2} \mathrm{O}$ which was then concentrated and purified by column chromatography (hexanes/ethyl acetate mixture). 


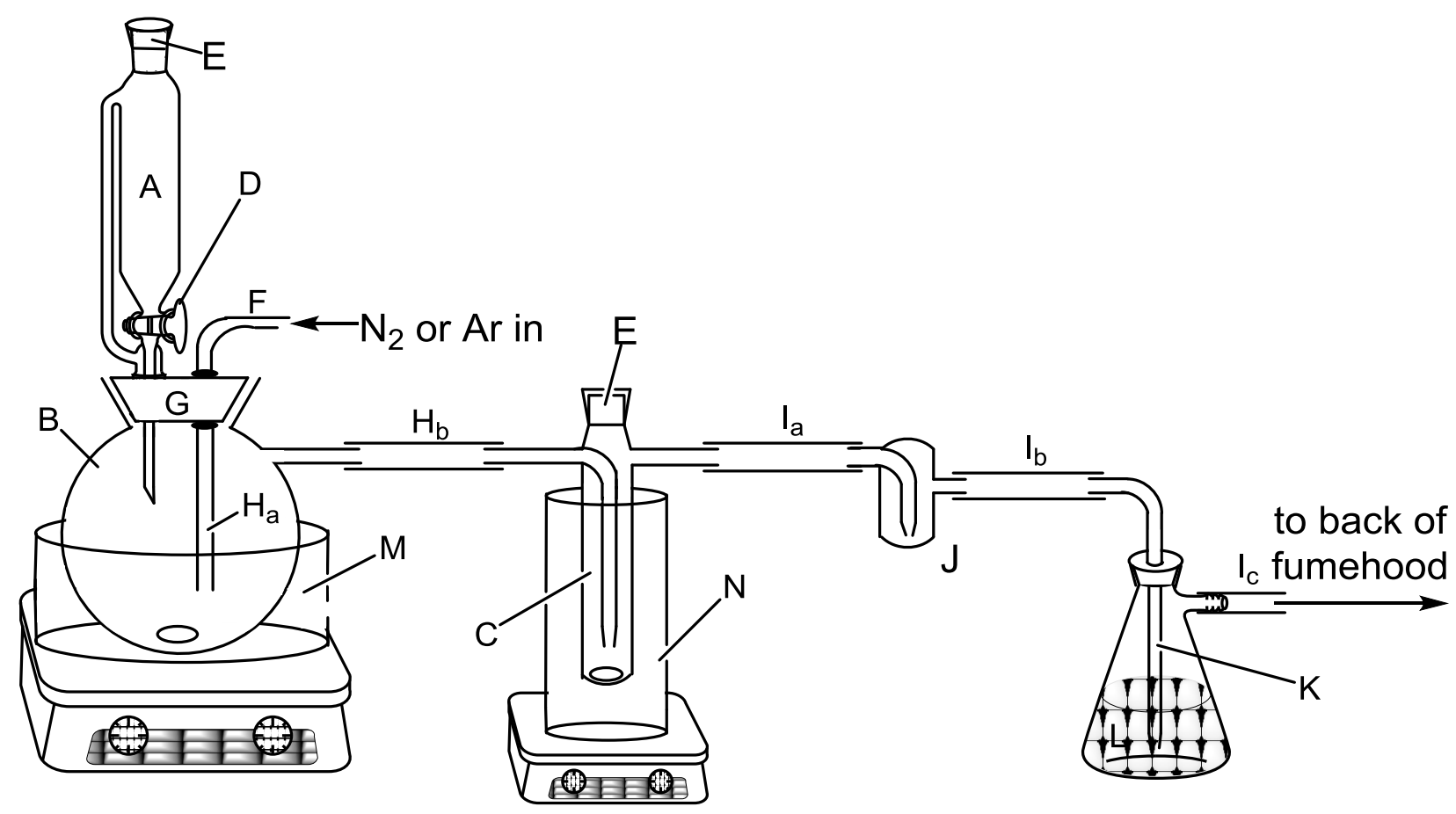

Figure S1. Experimental apparatus for the cyclopropanation of alkenes 1a-j, 3, and 5a-c.

A: Custom-made dropping funnel $(150 \mathrm{~mL}$ or $300 \mathrm{~mL}) . \mathrm{B}: 500 \mathrm{~mL}$ round bottom flask with side arm and wide neck equipped with extra-large stir bar. C: Reactor vessel (50 mL) with small hole at end of inlet tube for bubbles. D: Stopcock. E: Tightly fitted 24/40 rubber septum wired in place. F: Glass nitrogen/argon inlet (9mm OD). G: \#12 Rubber stopper with two 9mm bored holes. H: High density polyethylene tubing (7mm ID) with any cut sharp edges smoothed with a heat gun. I: Tygon ${ }^{\circledR}$ tubing. J: Empty bubbler as suck-back trap for AcOH bubbler. K: Glass inlet tube. L: Filter flask with AcOH: $\mathrm{H}_{2} \mathrm{O}$ (1:1) $\left(\mathrm{CH}_{2} \mathrm{~N}_{2}\right.$ trap and bubbler). M: Room temperature water bath. $\mathrm{N}: 0^{\circ} \mathrm{C}$ ice-water bath. $\quad$ Note: $\mathrm{OD}=$ outer diameter; ID = inner diameter. 


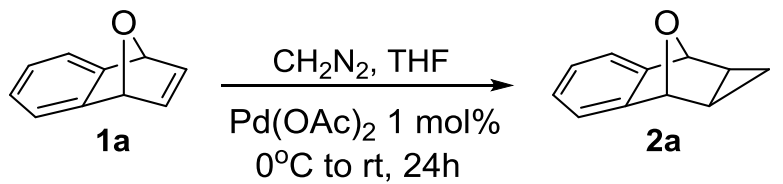

exo-cyclopropanated oxabenzonorbornadiene (2a) Yield $=2.45 \mathrm{~g}(90 \%)$; clear oil; $\mathrm{R}_{f}=0.52$ $($ EtOAc: hexanes $=1: 4)$. FTIR $\left(\mathrm{NaCl}, v, \mathrm{~cm}^{-1}\right): 3049,2999,1457,1054,955,834,755,644 .{ }^{1} \mathrm{H}$ NMR $\left(\mathrm{CDCl}_{3}, 400 \mathrm{MHz}\right): \delta 7.24(\mathrm{~m}, 2 \mathrm{H}), 7.09(\mathrm{~m}, 2 \mathrm{H}), 5.05(\mathrm{~s}, 2 \mathrm{H}), 1.50(\mathrm{td}, J=5.2,3.6 \mathrm{~Hz}$, $1 \mathrm{H}), 1.20(\mathrm{dd}, J=6.7,3.6 \mathrm{~Hz}, 2 \mathrm{H}), 0.86(\mathrm{td}, J=6.7,5.2,1 \mathrm{H}) .{ }^{13} \mathrm{C}$ NMR $\left(\mathrm{JMOD}, \mathrm{CDCl}_{3}, 100\right.$ MHz): $\delta 147.8,125.8,119.1,77.8,19.9,14.1$. Spectral data are consistent with those previously reported. $^{[15]}$

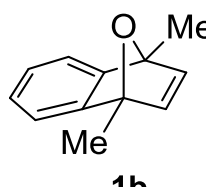

1b

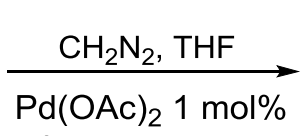

$0^{\circ} \mathrm{C}$ to $\mathrm{rt}, 12-24 \mathrm{~h}$

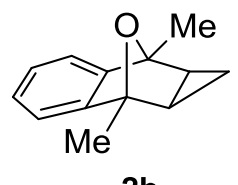

2b

exo-cyclopropanated oxabenzonorbornadiene (2b) Yield $=2.88 \mathrm{~g}(89 \%)$; white solid; $\mathrm{R}_{f}=$ 0.65 (EtOAc: hexanes = 1:4); m.p. $=38-40^{\circ} \mathrm{C}$. FTIR $\left(\mathrm{NaCl}, v, \mathrm{~cm}^{-1}\right): 3047,1450,1381,1280$, 1146, 862, 753. ${ }^{1} \mathrm{H}$ NMR $\left(\mathrm{CDCl}_{3}, 400 \mathrm{MHz}\right): \delta 7.18(\mathrm{~m}, 2 \mathrm{H}), 7.14(\mathrm{~m}, 2 \mathrm{H}), 1.73(\mathrm{~s}, 6 \mathrm{H}), 1.58$ $(\mathrm{dt}, J=5.0,3.4 \mathrm{~Hz}, 1 \mathrm{H}), 1.22(\mathrm{dd}, J=6.6,3.4 \mathrm{~Hz}, 2 \mathrm{H}), 0.81(\mathrm{td}, J=6.6,5.0,1 \mathrm{H}){ }^{13} \mathrm{C} \mathrm{NMR}$ $\left(\mathrm{JMOD}, \mathrm{CDCl}_{3}, 100 \mathrm{MHz}\right): \delta 151.0,125.7,117.7,83.5,26.6,15.5,13.8$. Spectral data are consistent with those previously reported. ${ }^{[15]}$

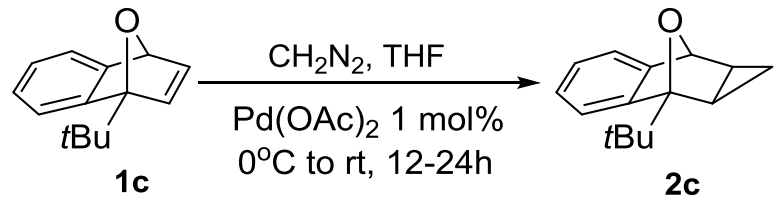

exo-cyclopropanated oxabenzonorbornadiene $(2 \mathrm{c})$ Yield $=1.37 \mathrm{~g}(85 \%)$; white solid; $\mathrm{R}_{f}=$ 0.59 (EtOAc: hexanes = 1:4); m.p. $=44-46^{\circ} \mathrm{C}$. FTIR $\left(\mathrm{NaCl}, v, \mathrm{~cm}^{-1}\right): 3047,2976,1459,1364$, 
1067, 923, 752. ${ }^{1} \mathrm{H}$ NMR $\left(\mathrm{CDCl}_{3}, 400 \mathrm{MHz}\right): \delta 7.43(\mathrm{~m}, 1 \mathrm{H}), 7.26(\mathrm{~m}, 1 \mathrm{H}), 7.10(\mathrm{~m}, 2 \mathrm{H}), 5.01$ $(\mathrm{s}, 1 \mathrm{H}), 1.56(\mathrm{dt}, J=5.2,3.6, \mathrm{~Hz}, 1 \mathrm{H}), 1.26(\mathrm{~s}, 9 \mathrm{H}), 1.24(\mathrm{td}, J=6.8,3.6 \mathrm{~Hz}, 1 \mathrm{H}), 1.16(\mathrm{td}, J=$ 6.8, 3.6 Hz, 1H), $0.96(\mathrm{td}, J=6.8,5.2 \mathrm{~Hz}, 1 \mathrm{H}) ;{ }^{13} \mathrm{C}$ NMR (JMOD, $\left.\mathrm{CDCl}_{3}, 100 \mathrm{MHz}\right): \delta 149.7$, $148.4,125.4,125.3,120.8,119.1,93.4,77.3,34.0,26.7,22.9,20.0,16.5$. Spectral data are consistent with those previously reported. ${ }^{[15]}$
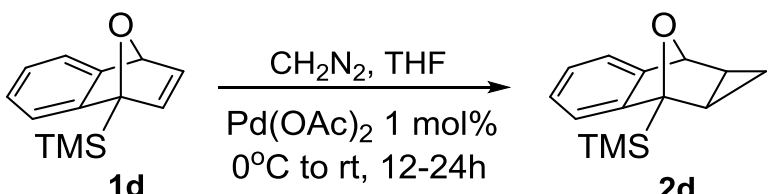

exo-cyclopropanated oxabenzonorbornadiene (2d) Yield $=799 \mathrm{mg}(75 \%)$; yellow solid; $\mathrm{R}_{f}=$ 0.33 (EtOAc: hexanes $=1: 19) ;$ m.p. $=35-36{ }^{\circ} \mathrm{C}$. FTIR $\left(\mathrm{KBr}, v, \mathrm{~cm}^{-1}\right): 3046,3001,2959,2900$, 1454, 1249, 1051, 840, 751; ${ }^{1} \mathrm{H}$ NMR (400 MHz, $\left.\mathrm{CDCl}_{3}\right): \delta$ 7.35-7.34 (m, 1H), 7.30-7.28 (m, 1H), 7.17-7.14 (m, 2H), $5.16(\mathrm{~s}, 1 \mathrm{H}), 1.50-1.47(\mathrm{~m}, 1 \mathrm{H}), 1.26-1.24(\mathrm{~m}, 2 \mathrm{H}), 0.99-0.94(\mathrm{~m}, 1 \mathrm{H})$, $0.36(\mathrm{~s}, 9 \mathrm{H}) ;{ }^{13} \mathrm{C} \mathrm{NMR}\left(100 \mathrm{MHz}, \mathrm{CDCl}_{3}\right): \delta 150.9,148.8,125.5,125.2,119.2,119.1,79.7,79.1$, 22.8, 20.2, 15.0, -2.9; HRMS: Calculated for $\mathrm{C}_{14} \mathrm{H}_{18} \mathrm{SiO}[\mathrm{M}]^{+}: 230.1127$. Found: 230.1122 .
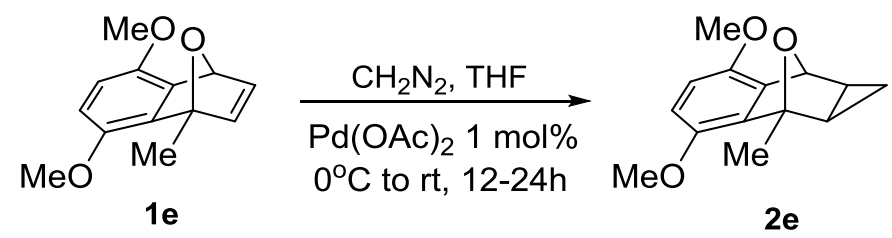

exo-cyclopropanated oxabenzonorbornadiene (2e) Yield $=1.74 \mathrm{~g}(82 \%)$; beige solid; $\mathrm{R}_{f}=$ 0.25 (EtOAc: hexanes = 1:9); m.p. $=132-134{ }^{\circ} \mathrm{C}$. FTIR $\left(\mathrm{KBr}, v, \mathrm{~cm}^{-1}\right): 3005,2983,2937,2834$, $1495,1436,1259,1043,809 ;{ }^{1} \mathrm{H} \operatorname{NMR}\left(400 \mathrm{MHz}, \mathrm{CDCl}_{3}\right): \delta 6.68\left(\mathrm{ABq}, J_{\mathrm{AB}}=8.8 \mathrm{~Hz}, \Delta \delta_{\mathrm{AB}}=\right.$ $9.8 \mathrm{~Hz}, 2 \mathrm{H}), 5.19$ (s, 1H), 3.80 (s, 3H), 3.79 (s, 3H), $1.86(\mathrm{~s}, 3 \mathrm{H}), 1.52-1.49(\mathrm{~m}, 1 \mathrm{H}), 1.38-1.33$ (m, 1H), 1.28-1.24 (m, 1H), 0.86-0.82 (m, 1H); $\left.{ }^{13} \mathrm{C} \mathrm{NMR} \mathrm{(100} \mathrm{MHz,} \mathrm{CDCl}_{3}\right): \delta$ 147.8, 146.9, 
138.2, 137.9, 111.2, 111.0, 85.4, 75.1, 56.1, 56.0, 23.9, 22.6, 16.8, 14.0; HRMS: Calculated for $\mathrm{C}_{14} \mathrm{H}_{16} \mathrm{O}_{3}[\mathrm{M}]^{+}:$232.1099. Found: 232.1092 .
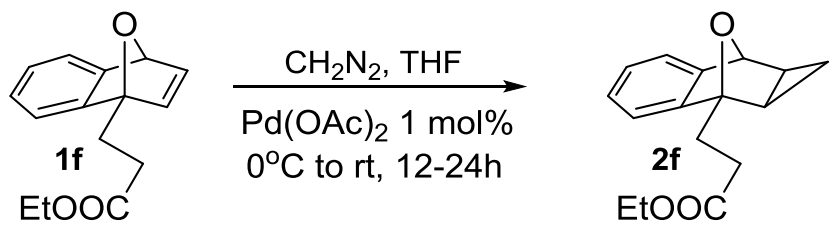

exo-cyclopropanated oxabenzonorbornadiene $(2 \mathrm{f})$ Yield $=1.28 \mathrm{~g}(81 \%)$; yellow oil; $\mathrm{R}_{f}=$ 0.23 (EtOAc: hexanes = 1:9); FTIR $\left(\mathrm{KBr}, v, \mathrm{~cm}^{-1}\right): 3048,2984,2932,1732,1314,1053,1034$; ${ }^{1} \mathrm{H}$ NMR $\left(400 \mathrm{MHz}, \mathrm{CDCl}_{3}\right): \delta$ 7.28-7.27 (m, 1H), 7.23-7.22 (m, 1H), 7.16-7.14 (m, 2H), $5.04(\mathrm{~d}$, $\mathrm{J}=2.3 \mathrm{~Hz}, 1 \mathrm{H}), 4.21-4.15(\mathrm{qd}, \mathrm{J}=7.2 \mathrm{~Hz}, 1.1 \mathrm{~Hz}, 2 \mathrm{H}), 2.67-2.51(\mathrm{~m}, 4 \mathrm{H}), 1.58-1.55(\mathrm{~m}, 1 \mathrm{H})$, 1.31-1.27 (m, 4H), 1.12-1.09 (m, 1H), 0.94-0.90 (m, 1H); ${ }^{13} \mathrm{C}$ NMR (100 MHz, $\left.\mathrm{CDCl}_{3}\right): \delta 173.63$, $148.9,148.6,125.9,119.3,118.2,86.3,77.5,60.4,29.5,25.2,22.5,22.3,14.7,14.3$; HRMS: Calculated for $\mathrm{C}_{16} \mathrm{H}_{18} \mathrm{O}_{3}[\mathrm{M}]^{+}:$258.1256. Found: 258.1251 .
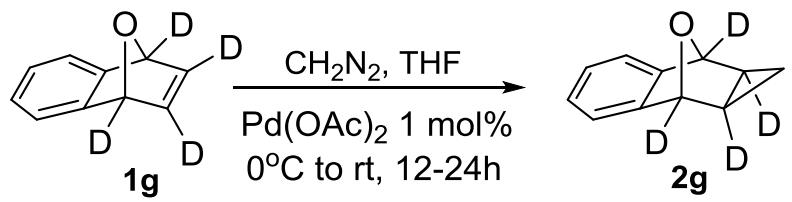

exo-cyclopropanated oxabenzonorbornadiene $(\mathbf{2 g})$ Yield = $930 \mathrm{mg}(85 \%)$; colorless oil; $\mathrm{R}_{f}=$ $0.24\left(\right.$ EtOAc: hexanes = 1:19); FTIR $\left(\mathrm{KBr}, \mathrm{v}, \mathrm{cm}^{-1}\right): 3075,3053,3003,2246,1457,1448,1193$, 1161, 1044, 952, 735; ${ }^{1} \mathrm{H}$ NMR (400 MHz, $\left.\mathrm{CDCl}_{3}\right): \delta$ 7.38-7.36 (m, 2H), 7.23-7.21 (m, 2H), 1.61 (m, 1H), $0.98(\mathrm{~m}, 1 \mathrm{H}) ;{ }^{13} \mathrm{C}$ NMR (100 MHz, $\left.\mathrm{CDCl}_{3}\right): \delta 148.0,126.0,119.3,77.3(\mathrm{t}, J=32 \mathrm{~Hz})$, $19.6(\mathrm{t}, J=26 \mathrm{~Hz}), 14.1 ;{ }^{2} \mathrm{H} \mathrm{NMR}\left(61.4 \mathrm{MHz}, \mathrm{CDCl}_{3}\right): 5.15$ (br s, 2H), 1.29 (br s, 2H); HRMS: Calculated for $\mathrm{C}_{11} \mathrm{H}_{6} \mathrm{D}_{4} \mathrm{O}[\mathrm{M}]^{+}:$: 162.0983. Found: 162.0986 . 


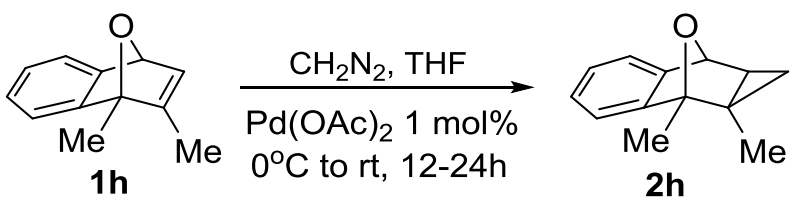

exo-cyclopropanated oxabenzonorbornadiene (2h) Yield = $510 \mathrm{mg}(94 \%)$; clear oil; $\mathrm{R}_{f}=0.23$ $($ EtOAc: hexanes $=1: 19)$. FTIR $\left(\mathrm{NaCl}, v, \mathrm{~cm}^{-1}\right): 3069,2973,2932,1453,1435,1385,1169,1132$, 1006. ${ }^{1} \mathrm{H} \mathrm{NMR}\left(\mathrm{CDCl}_{3}, 400 \mathrm{MHz}\right): \delta$ 7.38-7.35 (m, 1H), 7.32-7.30 (m, 1H), 7.27-7.21 (m, 2H), $5.05(\mathrm{~s}, 1 \mathrm{H}), 1.79-1.77(\mathrm{~m}, 1 \mathrm{H}), 1.78(\mathrm{~s}, 3 \mathrm{H}), 1.11-1.08(\mathrm{~m}, 1 \mathrm{H}), 1.05(\mathrm{~s}, 3 \mathrm{H}), 0.83-0.80(\mathrm{~m}, 1 \mathrm{H})$; ${ }^{13} \mathrm{C}$ NMR (JMOD, $\left.\mathrm{CDCl}_{3}, 100 \mathrm{MHz}\right): \delta$ 149.2, 148.4, 125.7, 125.4, 119.4, 119.0, 86.6, 77.1, 28.9, 28.4, 18.7, 14.6, 13.0. HRMS: Found: Calculated for $\mathrm{C}_{13} \mathrm{H}_{15} \mathrm{O}[\mathrm{M}+\mathrm{H}]^{+}$: 187.1117. Found: 187.1112.

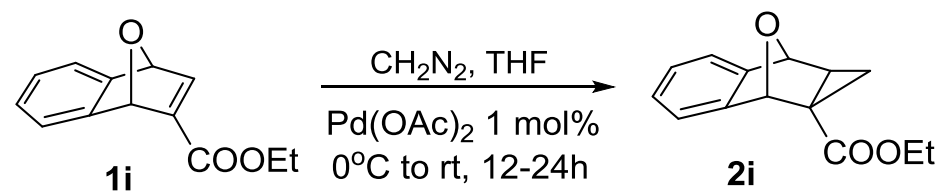

exo-cyclopropanated oxabenzonorbornadiene (2i) Yield = $380 \mathrm{mg}(72 \%)$; yellow solid; $\mathrm{R}_{f}=$ 0.49 (EtOAc: hexanes = 1:1); m.p. $=60-62{ }^{\circ} \mathrm{C}$. FTIR $\left(\mathrm{NaCl}, v, \mathrm{~cm}^{-1}\right): 3052,2983,2934,1721$, 1374, 1279, 1241, 1140, 759; ${ }^{1} \mathrm{H}$ NMR $\left(\mathrm{CDCl}_{3}, 400 \mathrm{MHz}\right): \delta$ 7.45-7.44 (m, 1H), 7.32-7.31 (m, 1H), 7.19-7.12 (m, 2H), $5.29(\mathrm{~s}, 1 \mathrm{H}), 5.11(\mathrm{~s}, 1 \mathrm{H}), 4.10-3.99(\mathrm{~m}, 2 \mathrm{H}), 2.14(\mathrm{t}, J=4.7 \mathrm{~Hz}, 1 \mathrm{H})$, 1.96-1.93 (m, 1H), 1.80-1.77 (m, 1H), $1.23(\mathrm{t}, J=7.1 \mathrm{~Hz}, 3 \mathrm{H}) ;{ }^{13} \mathrm{C}$ NMR (JMOD, $\mathrm{CDCl}_{3}, 100$ MHz): $\delta 172.0,147.0,146.9,126.2,126.1,122.0,119.4,78.4,77.6,60.6,34.4,33.1,22.7,14.2$; HRMS: Calculated for $\mathrm{C}_{14} \mathrm{H}_{14} \mathrm{O}_{3}[\mathrm{M}]^{+}:$230.0943. Found: 230.0940 . 


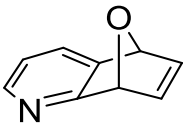

$1 \mathbf{j}$

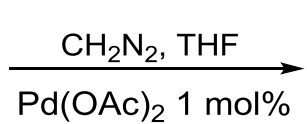

$0^{\circ} \mathrm{C}$ to rt, $12-24 \mathrm{~h}$

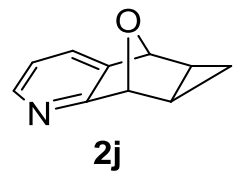

2j

exo-cyclopropanated oxabenzonorbornadiene $(\mathbf{2} \mathbf{j})$ Yield $=140 \mathrm{mg}(65 \%)$; beige solid; $\mathrm{R}_{f}=$ 0.28 (EtOAc: hexanes = 1:1); m.p. $=45-47^{\circ} \mathrm{C}$. FTIR $\left(\mathrm{NaCl}, v, \mathrm{~cm}^{-1}\right): 3054,3043,3002,2927$, 1578, 1400, 1258, 952, 830, 642; ${ }^{1} \mathrm{H} \mathrm{NMR}\left(\mathrm{CDCl}_{3}, 400 \mathrm{MHz}\right): \delta 8.20$ (dd, J=10.6 Hz, $1.2 \mathrm{~Hz}$, 1H), 7.52 (dd, $J=14.6 \mathrm{~Hz}, 1.1 \mathrm{~Hz}, 1 \mathrm{H}), 7.01$ (dd, $J=14.6 \mathrm{~Hz}, 5.3 \mathrm{~Hz}, 1 \mathrm{H}), 5.11(\mathrm{~s}, 1 \mathrm{H}), 5.06$ (s, $1 \mathrm{H}), 1.61-1.59(\mathrm{~m}, 1 \mathrm{H}), 1.41-1.7(\mathrm{~m}, 1 \mathrm{H}), 1.32-1.28(\mathrm{~m}, 1 \mathrm{H}), 1.01-0.97(\mathrm{~m}, 1 \mathrm{H}) ;{ }^{13} \mathrm{C}$ NMR $\left(\mathrm{JMOD}, \mathrm{CDCl}_{3}, 100 \mathrm{MHz}\right): \delta$ 169.3, 145.4, 141.0, 126.5, 120.5, 77.8, 77.1, 20.4, 18.4, 14.3; HRMS: Calculated for $\mathrm{C}_{10} \mathrm{H}_{9} \mathrm{NO}[\mathrm{M}]^{+}:$159.0684. Found: 159.0689.
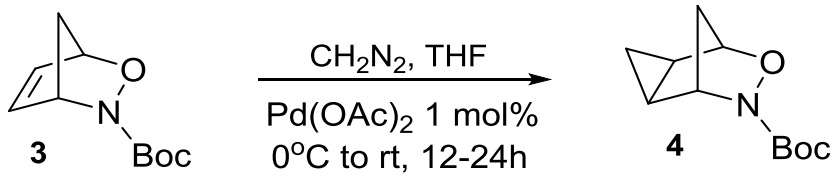

exo-6-oxa-7-N-butoxycarbonyltricyclo[3.2.1.0 $\left.{ }^{2,4}\right]$ oxazine (4) Yield = $907 \mathrm{mg}(97 \%)$; white solid; $\mathrm{R}_{f}=0.25($ EtOAc: hexanes $=1: 4) ; \mathrm{m} . \mathrm{p} .=72-74{ }^{\circ} \mathrm{C}\left(\right.$ lit. $\left.83-85{ }^{\circ} \mathrm{C}\right) ;$ FTIR $\left(\mathrm{KBr}, \mathrm{v}, \mathrm{cm}^{-1}\right)$ : 2982, 2252, $1722(\mathrm{C}=\mathrm{O}), 1478,1393,1163,909,734 ;{ }^{1} \mathrm{H}$ NMR $\left(400 \mathrm{MHz}, \mathrm{CDCl}_{3}\right): \delta 4.78(\mathrm{~m}$, 1H), $4.56(\mathrm{~m}, 1 \mathrm{H}), 1.56(\mathrm{~d}, J=18.3 \mathrm{~Hz}, 1 \mathrm{H}), 1.50(\mathrm{~s}, 9 \mathrm{H}), 1.45-1.38(\mathrm{~m}, 3 \mathrm{H}), 0.39-0.33(\mathrm{~m}, 2 \mathrm{H})$; ${ }^{13} \mathrm{C} \mathrm{NMR}\left(100 \mathrm{MHz}, \mathrm{CDCl}_{3}\right): \delta 157.8,81.9,80.7,60.8,28.3,27.7,13.9,12.9,4.1$; Spectral data are consistent with those previously reported. ${ }^{[13]}$ 


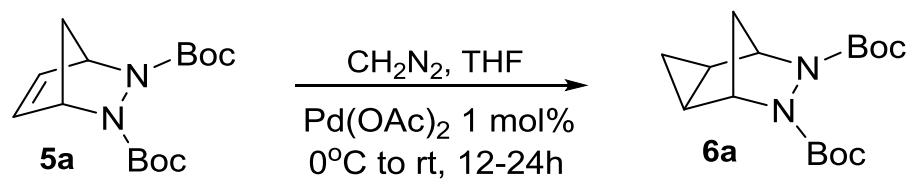

exo-6-oxa-7-N-butoxycarbonyltricyclo[3.2.1.0 $\left.{ }^{2,4}\right]$ hydrazine (6a) Yield = $516 \mathrm{mg}(95 \%)$; white solid; $\mathrm{R}_{f}=0.08$ (EtOAc: hexanes $\left.=1: 9\right) ;$ m.p. $=117-119{ }^{\circ} \mathrm{C}$. FTIR $\left(\mathrm{KBr}, v, \mathrm{~cm}^{-1}\right): 2977$, 2932, 1737, 1694 (C=O), 1367, 1339, 1164, 1111; ${ }^{1} \mathrm{H}$ NMR (400 MHz, $\left.\mathrm{CDCl}_{3}\right): 4.68$ (br s, 1H), 4.42 (br s, 1H), $1.59(\mathrm{~m}, 1 \mathrm{H}), 1.43(\mathrm{~s}, 18 \mathrm{H}), 1.31$ (d, J=11.3 Hz, 1H), 1.20-1.06 (m, 2H), 0.42$0.39(\mathrm{~m}, 1 \mathrm{H}), 0.33-0.28(\mathrm{~m}, 1 \mathrm{H}) ;{ }^{13} \mathrm{C} \mathrm{NMR}\left(125 \mathrm{MHz}, \mathrm{CDCl}_{3}\right): \delta[156.9,156.3,155.3](\mathrm{C}=\mathrm{O}$; peaks appear split due to relative orientation about nitrogen in different invertomers) ${ }^{[31]}[80.2$, 80.0], [61.3, 60.4, 59.6], 27.1, 25.8, [13.4, 13.0, 11.6, 11.1], 3.6; HRMS: Calculated for $\mathrm{C}_{16} \mathrm{H}_{26} \mathrm{~N}_{2} \mathrm{O}_{4}[\mathrm{M}]^{+}:$310.1893. Found: 310.1890 .
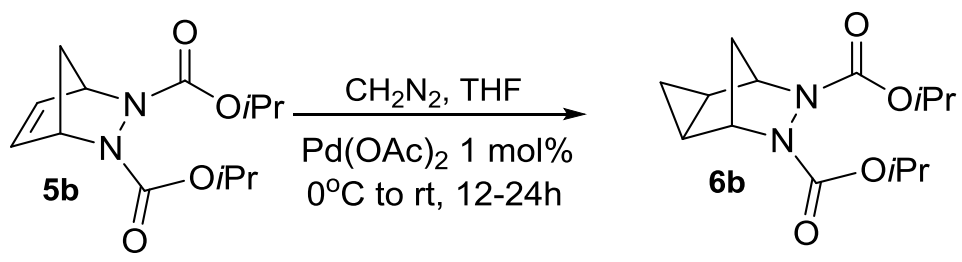

exo-6-oxa-7-N-isopropoxycarbonyltricyclo $\left[3.2 .1 .0^{2,4}\right]$ hydrazine (6b) Yield = $803 \mathrm{mg}(94 \%)$; white solid; $\mathrm{R}_{f}=0.27$ (EtOAc: hexanes $\left.=1: 4\right) ;$ m.p. $=55-57{ }^{\circ} \mathrm{C} ;$ FTIR $\left(\mathrm{KBr}, \mathrm{v}, \mathrm{cm}^{-1}\right): 2980$, 2938, 1739, 1694, 1468, 1373, 1339, 1104; ${ }^{1} \mathrm{H}$ NMR (400 MHz, $\left.\mathrm{CDCl}_{3}\right): \delta 4.71(\mathrm{~s}, 1 \mathrm{H}), 4.45$ (s, 1H), 1.33 (d, $J=11.4 \mathrm{~Hz}, 2 \mathrm{H}), 1.22$ (br s, 12H), 1.08 (d, J=8.6 Hz, 2H), 0.43-0.40 (m, 1H), 0.34$0.28(\mathrm{~m}, 1 \mathrm{H}) ;{ }^{13} \mathrm{C} \mathrm{NMR}\left(125 \mathrm{MHz}, \mathrm{CDCl}_{3}\right): \delta[157.9,157.5,157.2](\mathrm{C}=\mathrm{O}$; peaks appear split due to relative orientation about nitrogen in different invertomers) ${ }^{[31]}[69.7,69.5],[62.0,61.1]$, 26.5, 21.7, [13.9, 12.2], 4.3; HRMS: Calculated for $\mathrm{C}_{14} \mathrm{H}_{22} \mathrm{~N}_{2} \mathrm{O}_{4}[\mathrm{M}]^{+}$: 282.1580. Found: 282.1585 . 


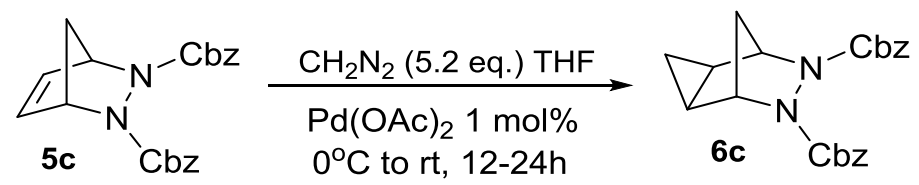

exo-6-oxa-7-N-carboxybenzyltricyclo[3.2.1.0 $\left.{ }^{\mathbf{2}, 4}\right]$ hydrazine $(6 \mathrm{c})$ Yield = $629 \mathrm{mg}(98 \%)$; colourless oil; $\mathrm{R}_{f}=0.15($ EtOAc: hexanes $=3: 17)$. FTIR $\left(\mathrm{KBr}, v, \mathrm{~cm}^{-1}\right): 3032,1739,1703,1455$, 1388, 1321, 1267, 1191, 1111; ${ }^{1} \mathrm{H}$ NMR (400 MHz, $\left.\mathrm{CDCl}_{3}\right): \delta 7.34$ (br s, 10H), 5.23 (m, 4H), $4.84(\mathrm{~s}, 1 \mathrm{H}), 4.60(\mathrm{~s}, 1 \mathrm{H}), 1.72-1.18(\mathrm{~m}, 4 \mathrm{H}), 0.47$ (br s, $1 \mathrm{H}), 0.38(\mathrm{~m}, 1 \mathrm{H}) ;{ }^{13} \mathrm{C} \mathrm{NMR}(125 \mathrm{MHz}$, $\left.\mathrm{CDCl}_{3}\right): \delta[158.3,157.8](\mathrm{C}=\mathrm{O}$; peaks appear split due to relative orientation about nitrogen in different invertomers), ${ }^{[31]} 135.8,128.3,127.9,127.7,67.7,[62.6,61.6], 26.8,[14.1,12.0], 4.5$; HRMS: Calculated for $\mathrm{C}_{22} \mathrm{H}_{22} \mathrm{~N}_{2} \mathrm{O}_{4}[\mathrm{M}]^{+}:$378.1580. Found: 378.1583 . 

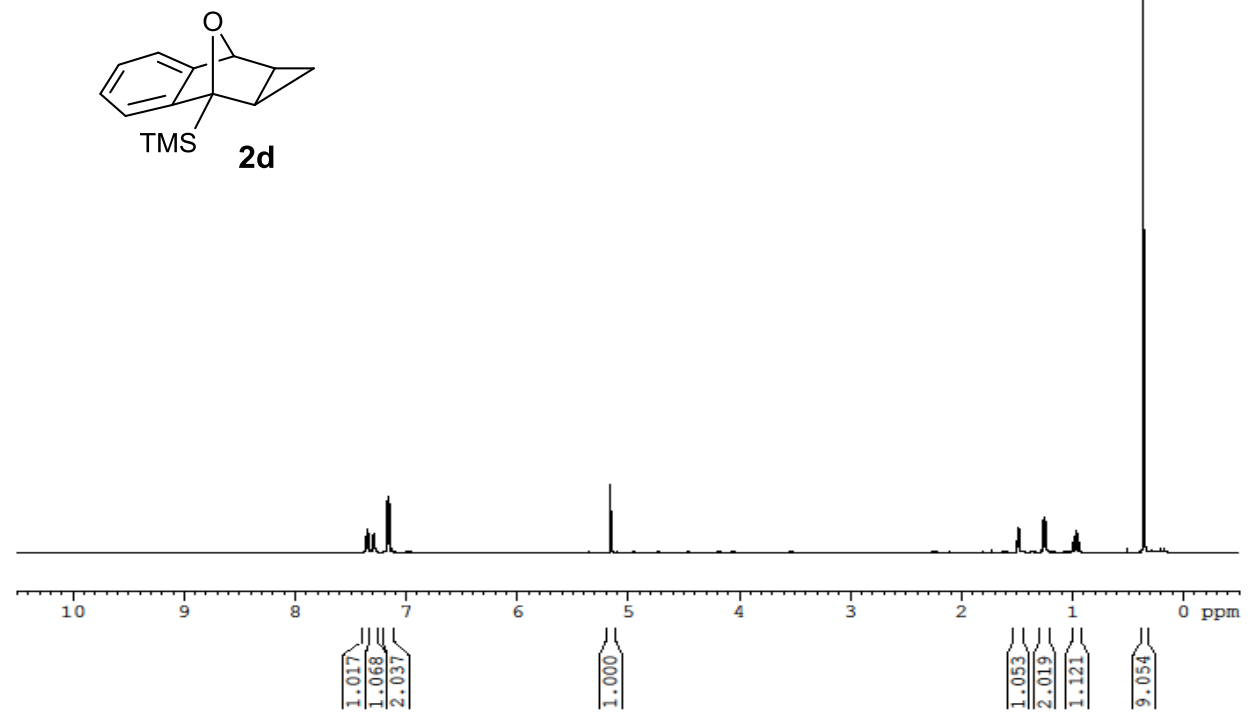

Figure S2. $400 \mathrm{MHz}^{1} \mathrm{H}$ NMR spectrum of cyclopropane $2 \mathrm{~d}$ in $\mathrm{CDCl}_{3}$

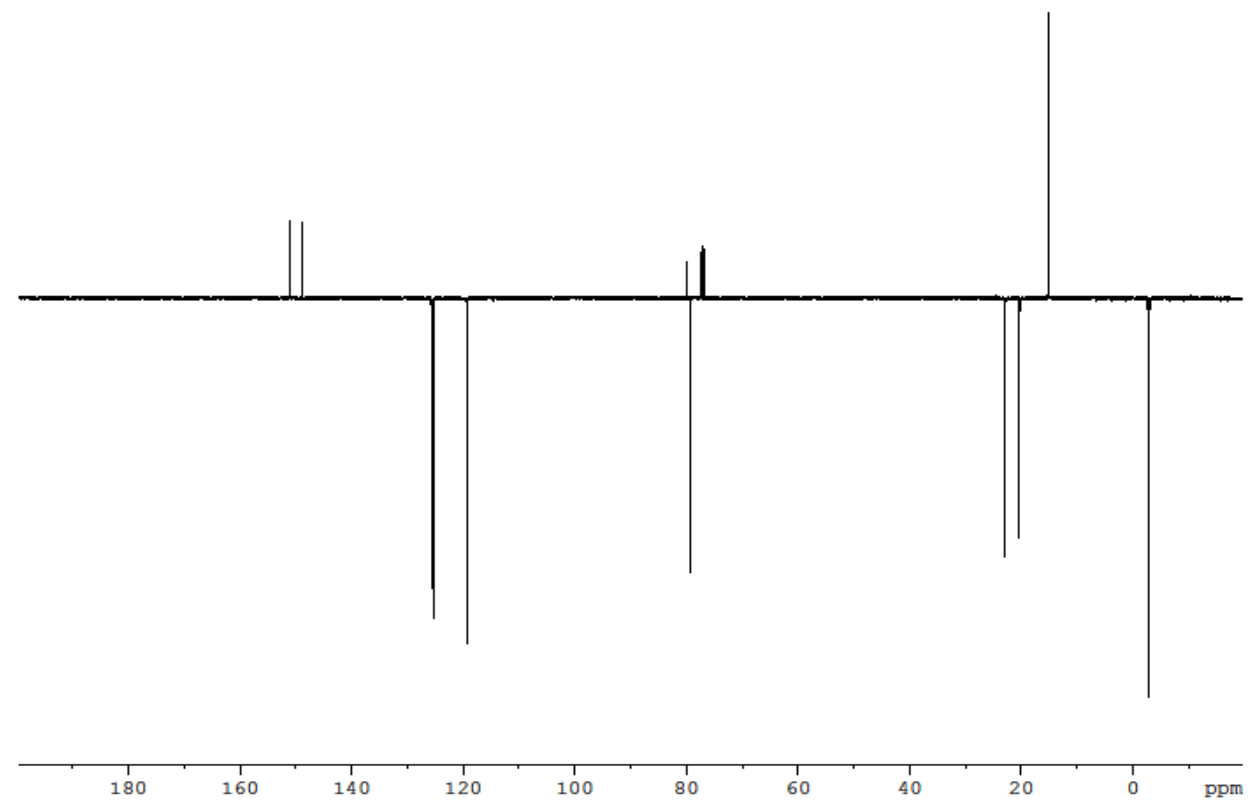

Figure S3. $100 \mathrm{MHz}^{13} \mathrm{C} \mathrm{NMR}$ spectrum of cyclopropane $2 \mathrm{~d}$ in $\mathrm{CDCl}_{3}$ 


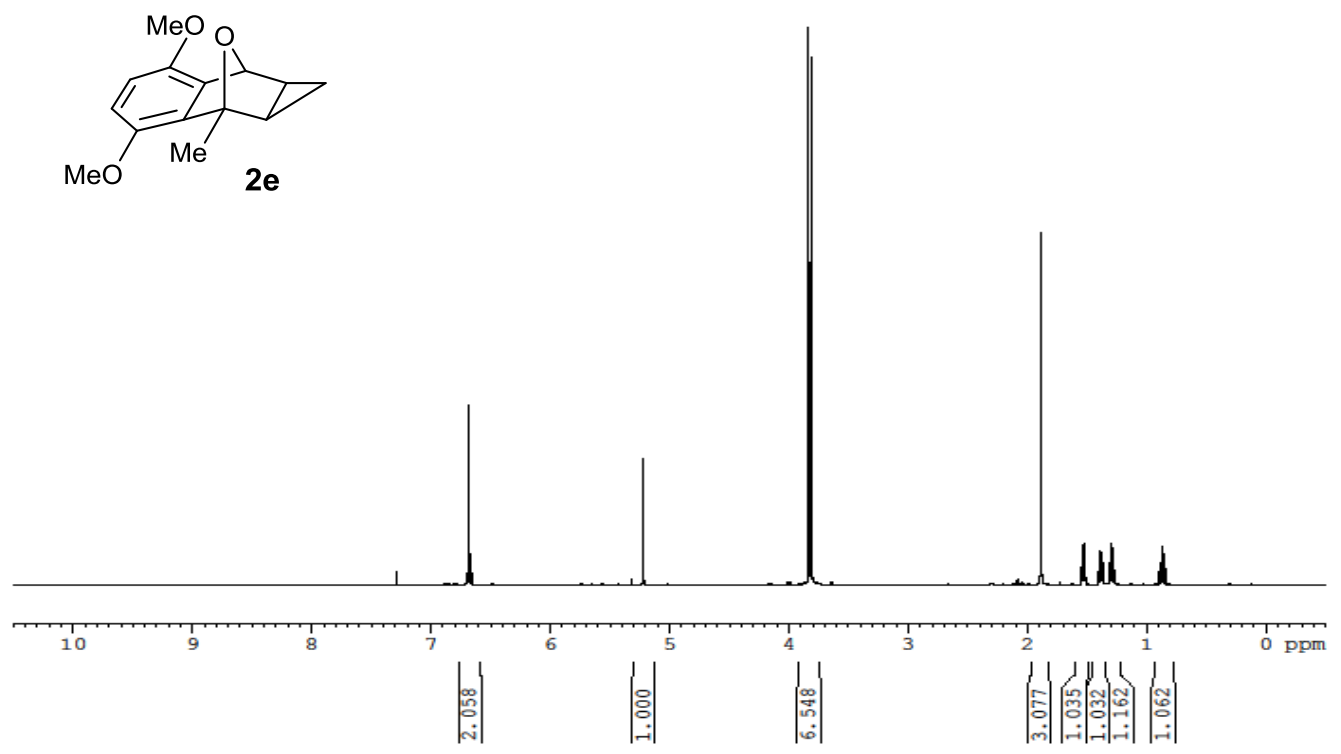

Figure S4. $400 \mathrm{MHz}^{1} \mathrm{H}$ NMR spectrum of cyclopropane $2 \mathrm{e}$ in $\mathrm{CDCl}_{3}$

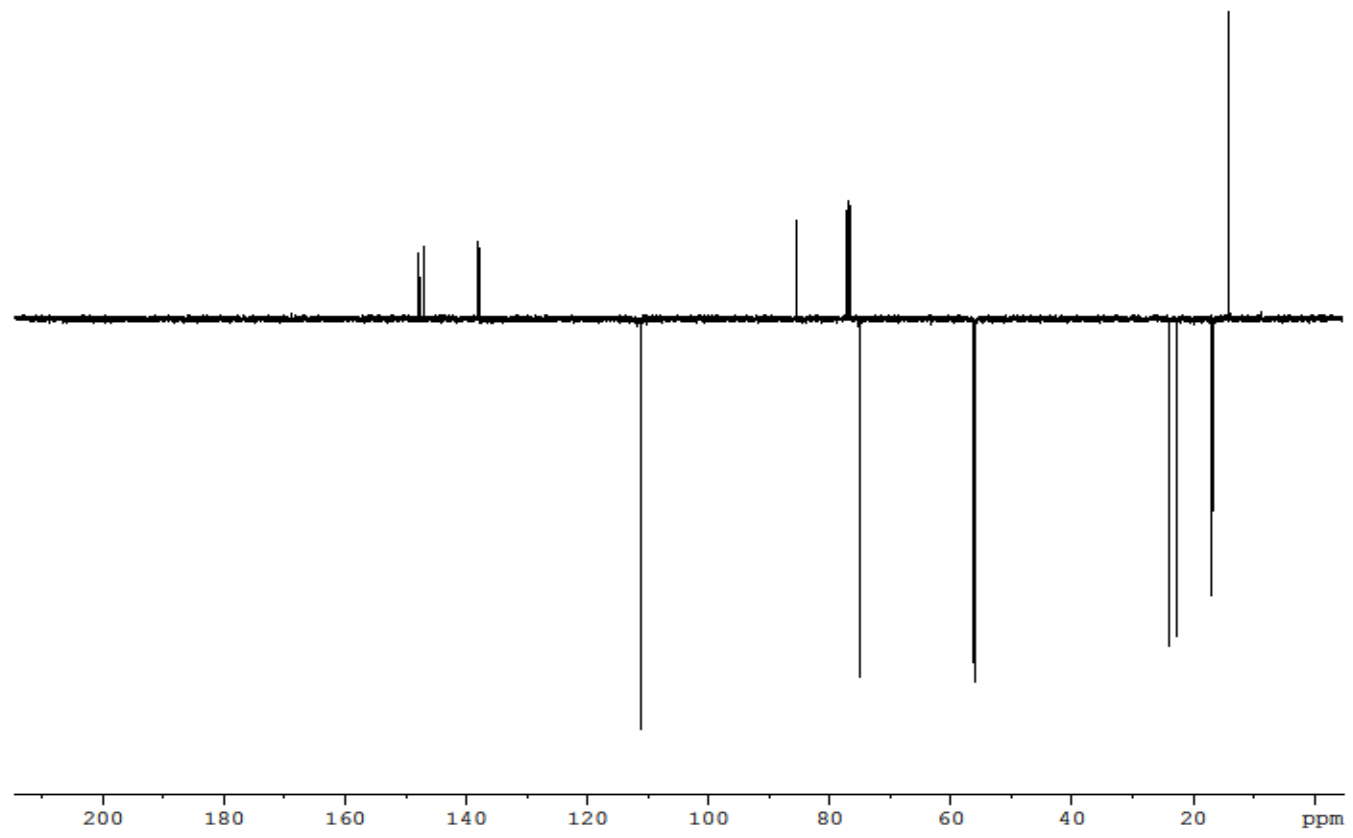

Figure S5. $100 \mathrm{MHz}^{13} \mathrm{C} \mathrm{NMR}$ spectrum of cyclopropane $2 \mathrm{e}$ in $\mathrm{CDCl}_{3}$ 

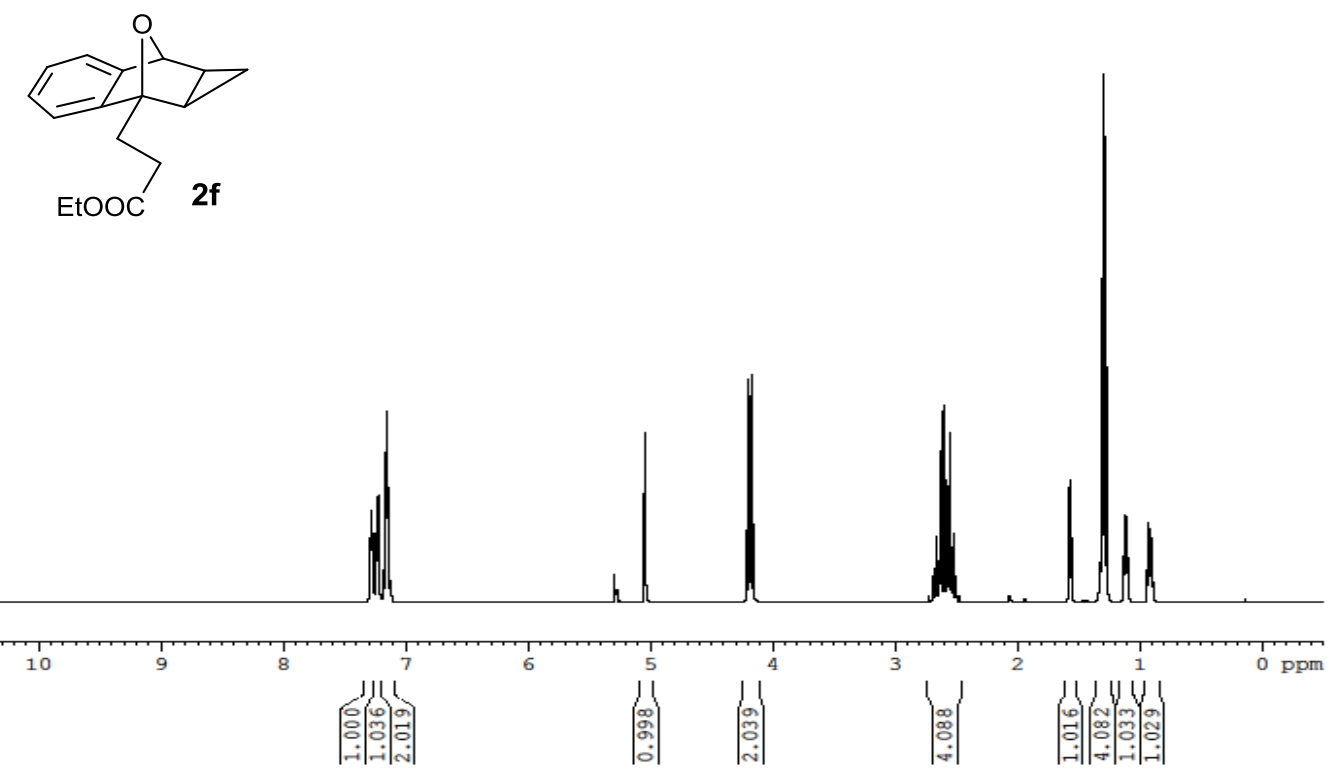

Figure S6. $400 \mathrm{MHz}{ }^{1} \mathrm{H}$ NMR spectrum of cyclopropane $2 \mathrm{f}$ in $\mathrm{CDCl}_{3}$

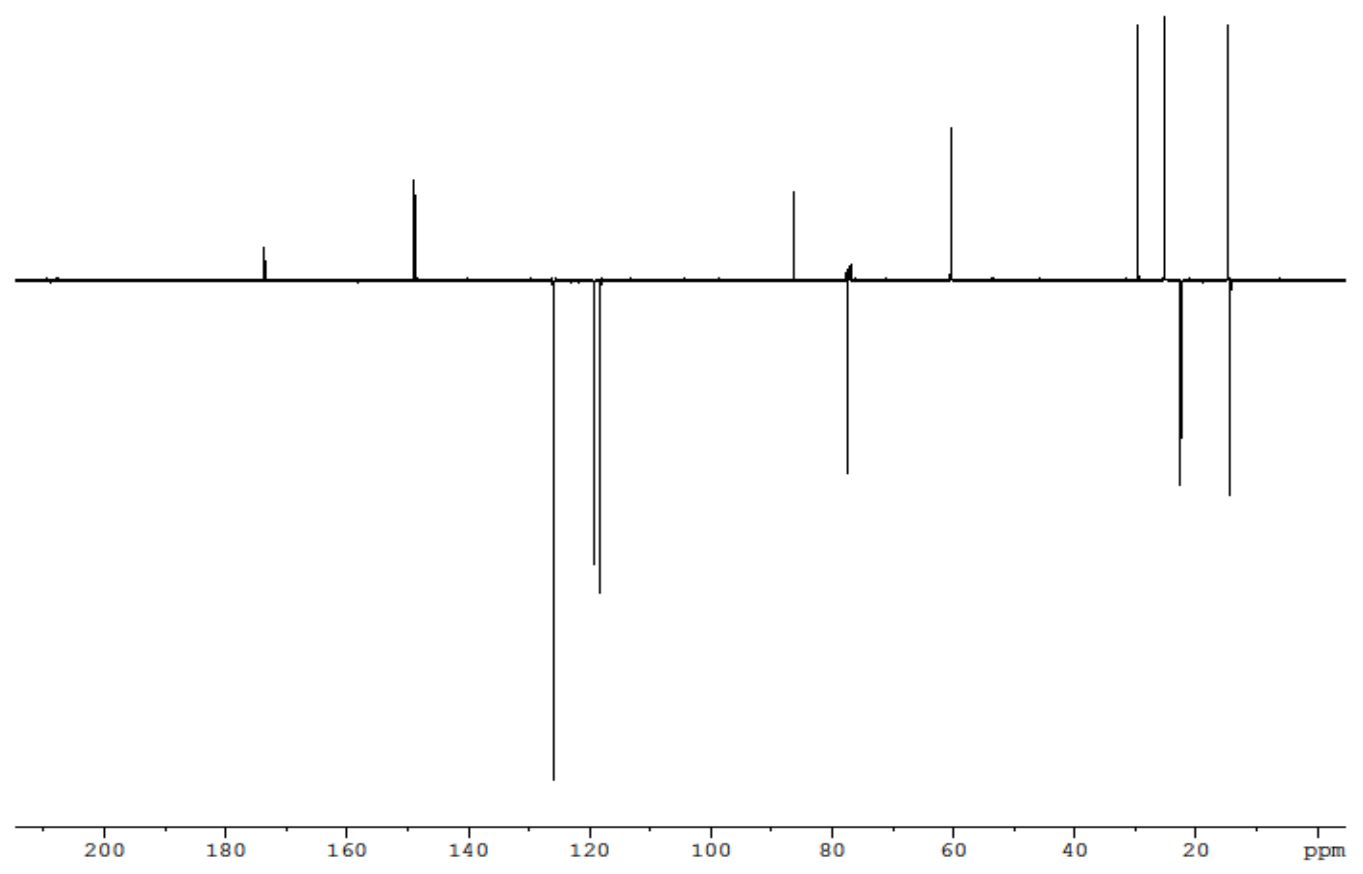

Figure $\mathrm{S7} .100 \mathrm{MHz}^{13} \mathrm{C}$ NMR spectrum of cyclopropane $2 \mathrm{f}$ in $\mathrm{CDCl}_{3}$ 


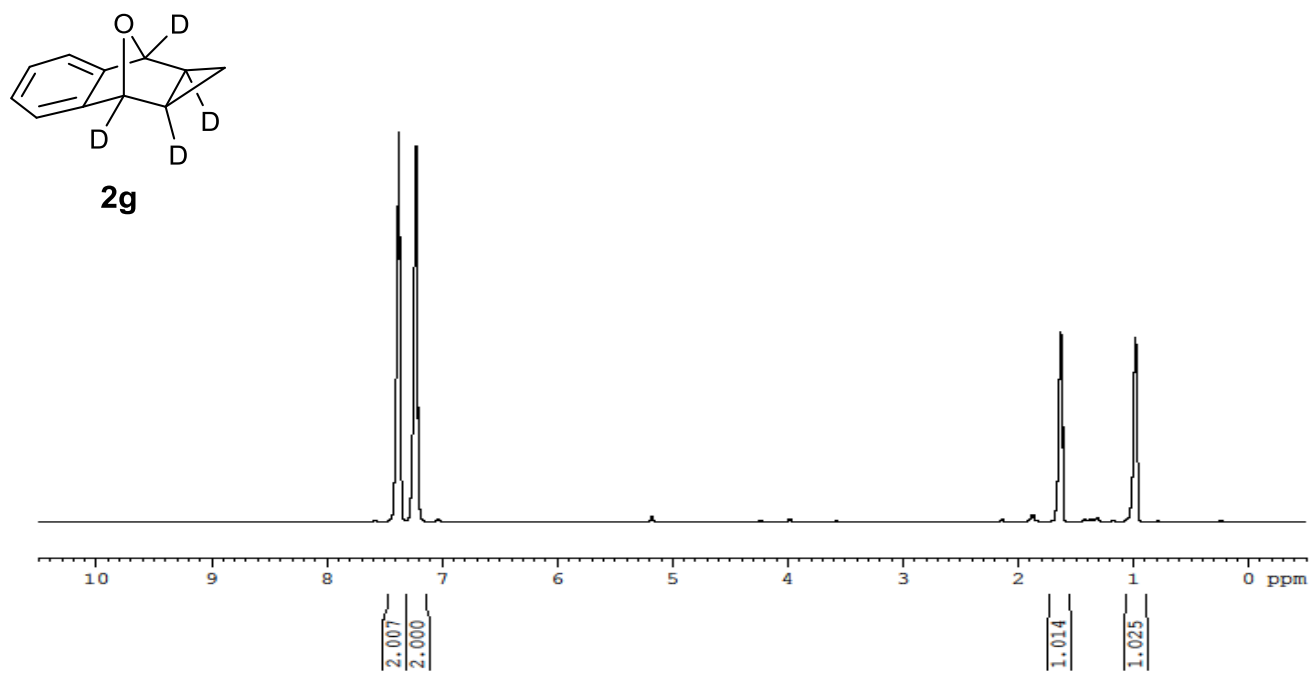

Figure S8. $400 \mathrm{MHz}^{1} \mathrm{H}$ NMR spectrum of cyclopropane $2 \mathrm{~g}$ in $\mathrm{CDCl}_{3}$

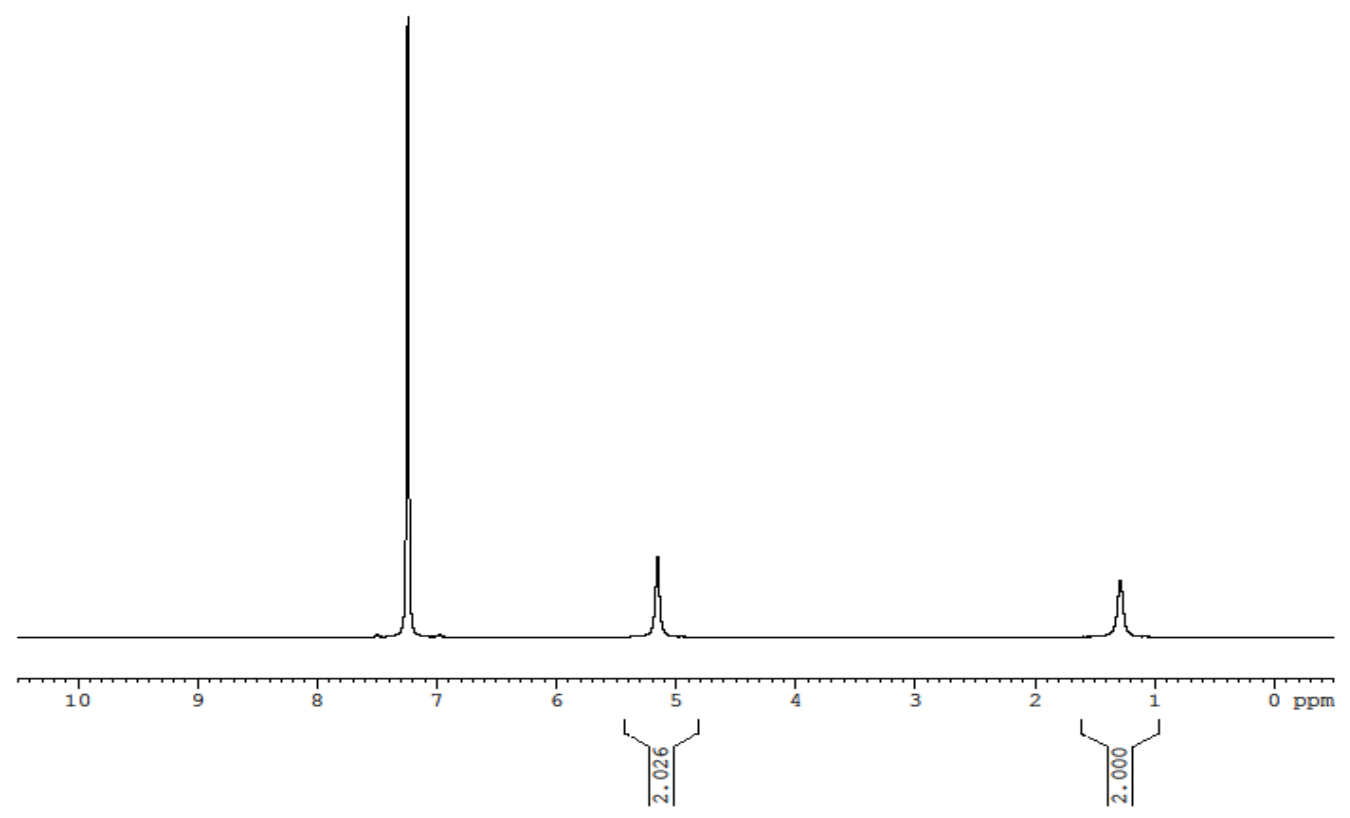

Figure S9. 61.4 $\mathrm{MHz}^{2} \mathrm{H}$ NMR spectrum of cyclopropane 2g in $\mathrm{CDCl}_{3}$ 


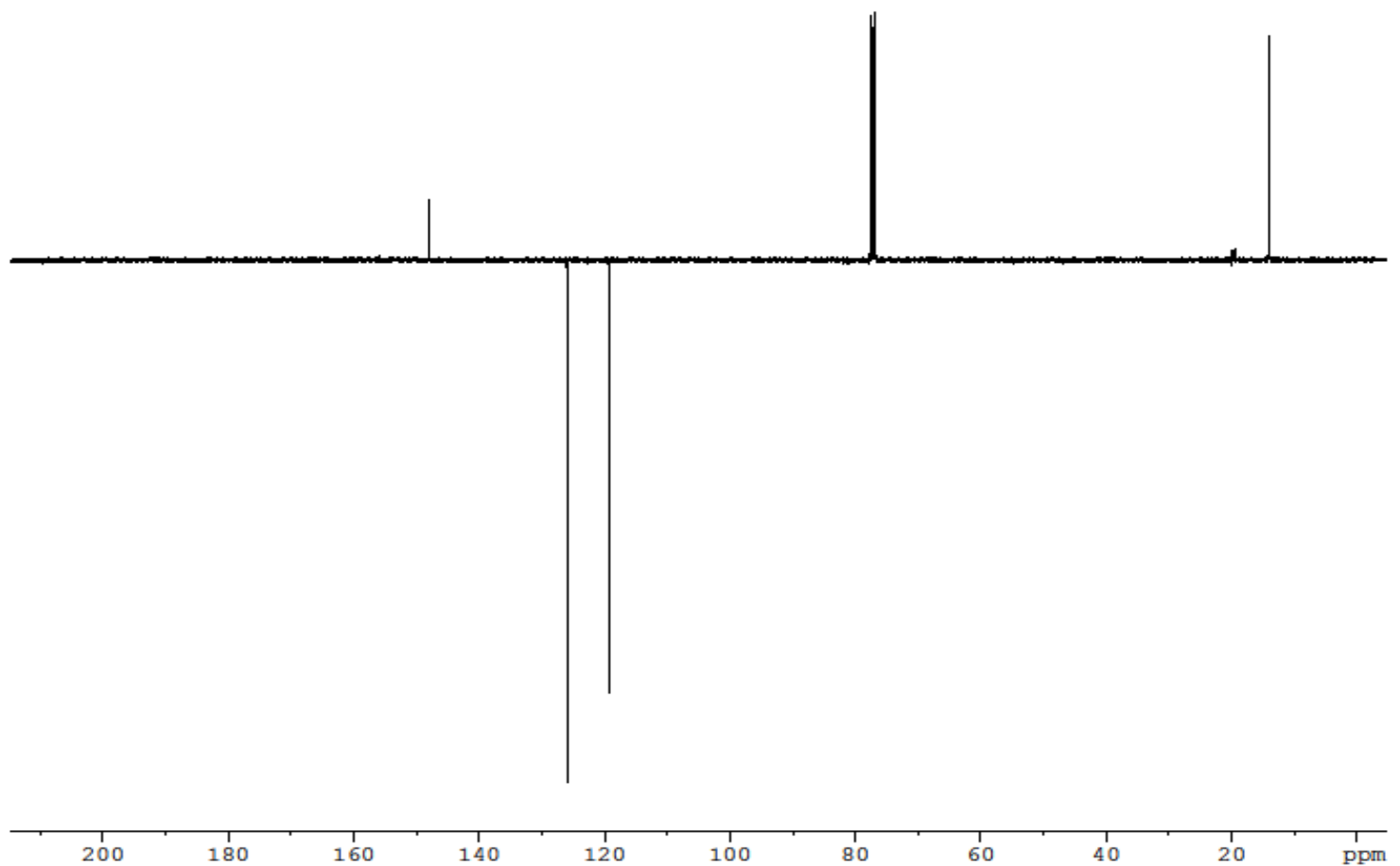

Figure S10. $100 \mathrm{MHz}{ }^{13} \mathrm{C} \mathrm{NMR}$ spectrum of cyclopropane $2 \mathrm{~g}$ in $\mathrm{CDCl}_{3}$ 


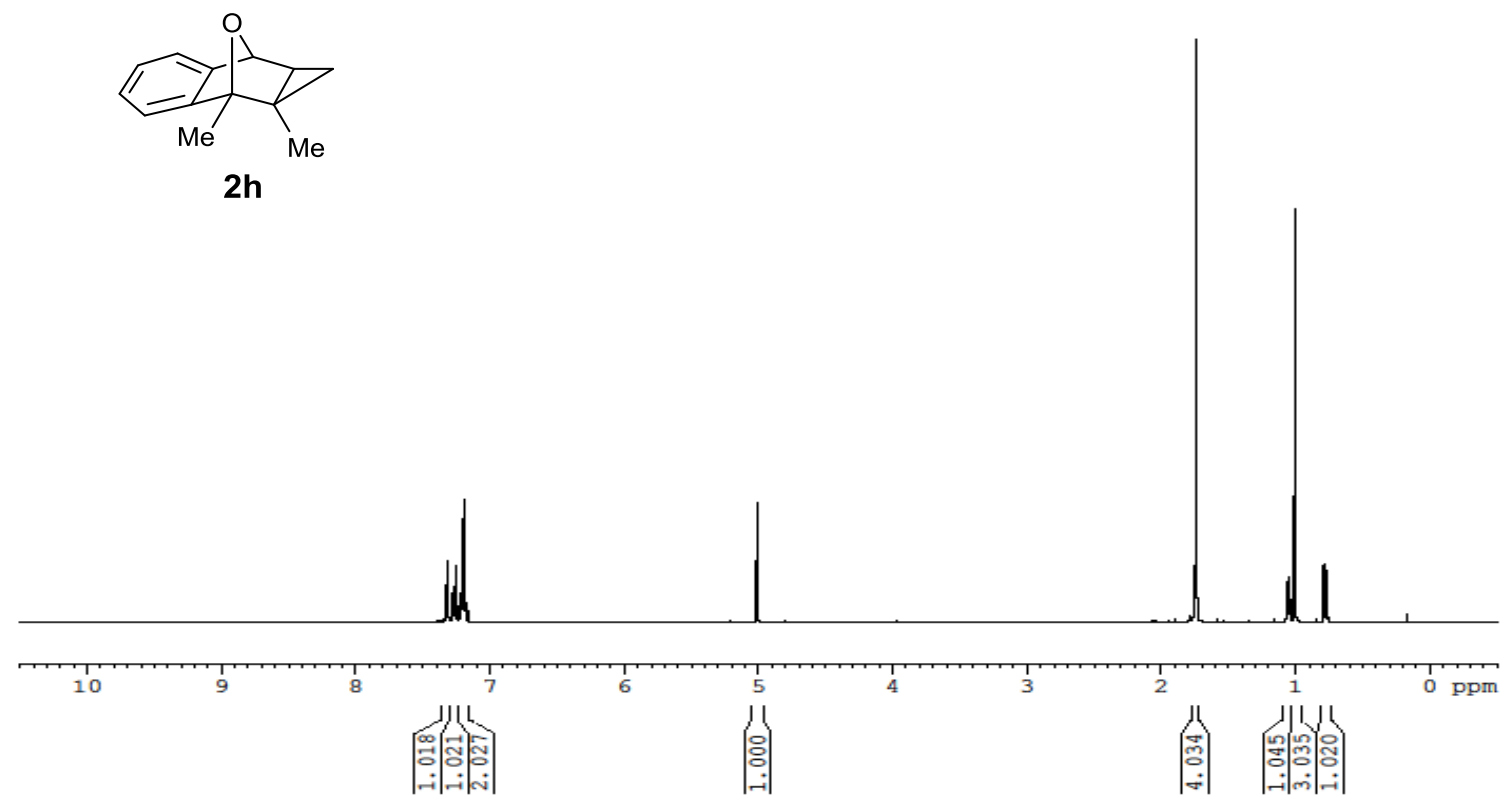

Figure S11. $400 \mathrm{MHz}^{1} \mathrm{H}$ NMR spectrum of cyclopropane $2 \mathrm{~h}$ in $\mathrm{CDCl}_{3}$

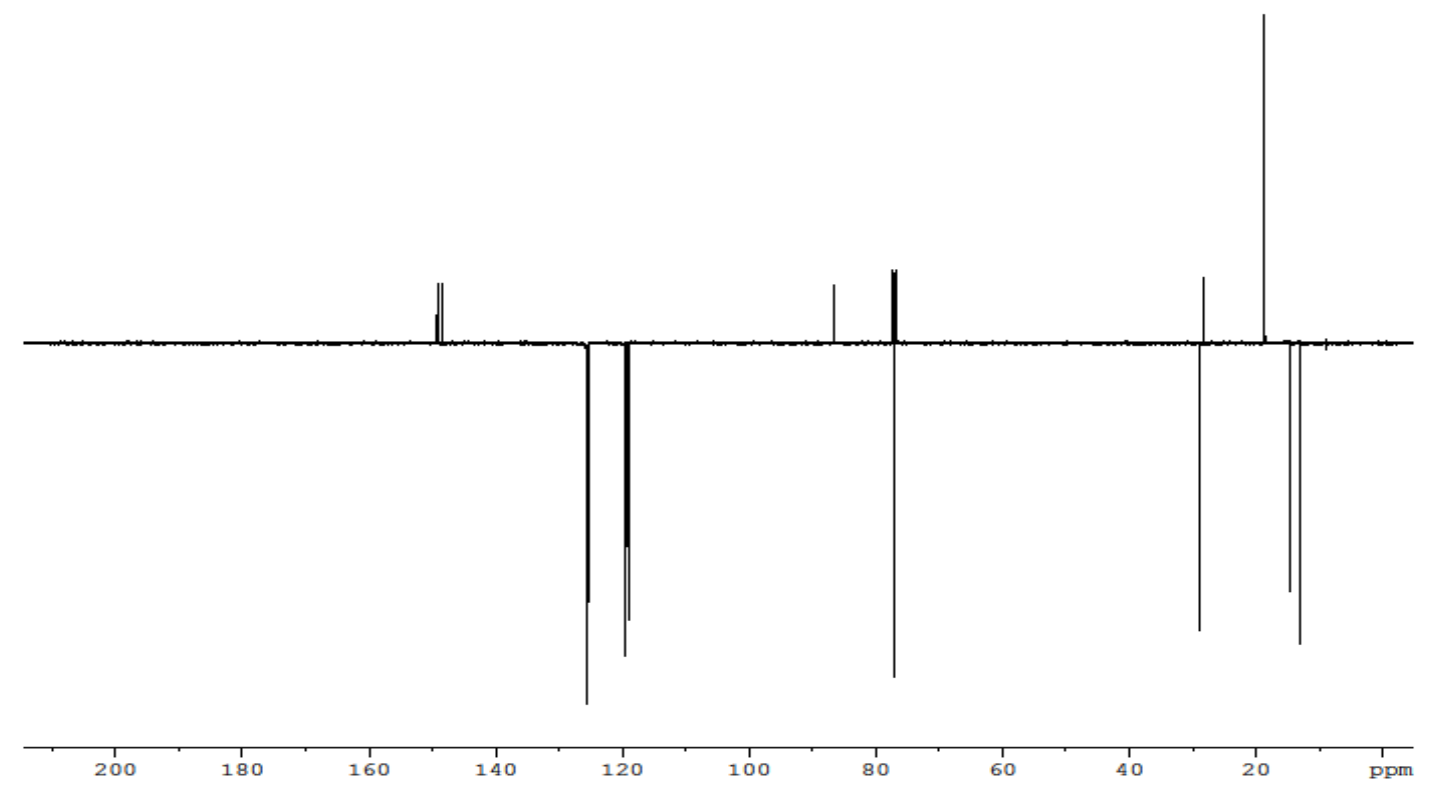

Figure S12. $100 \mathrm{MHz}^{13} \mathrm{C}$ NMR spectrum of cyclopropane $2 \mathrm{~h}$ in $\mathrm{CDCl}_{3}$ 

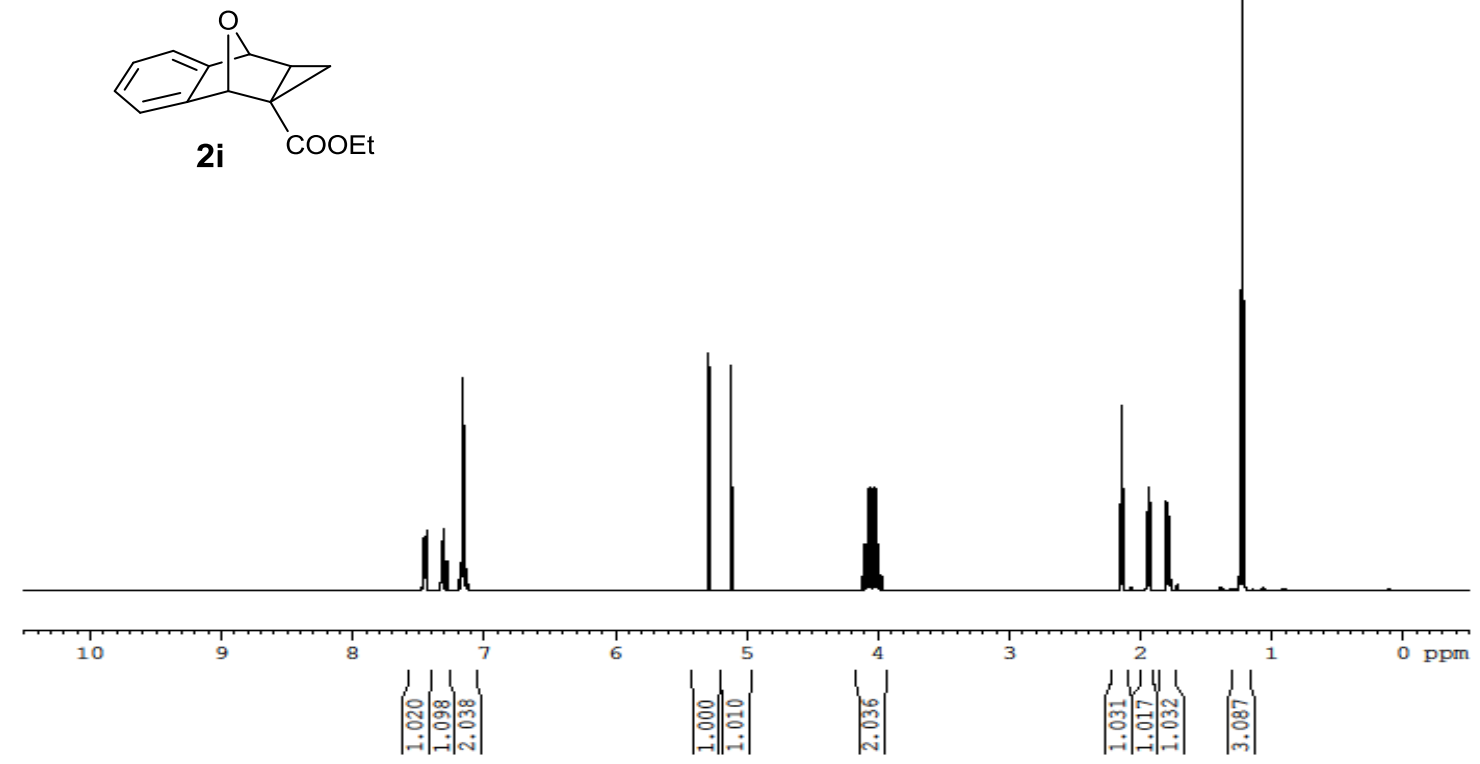

Figure S13. $400 \mathrm{MHz}^{1} \mathrm{H}$ NMR spectrum of cyclopropane $2 \mathrm{i}$ in $\mathrm{CDCl}_{3}$

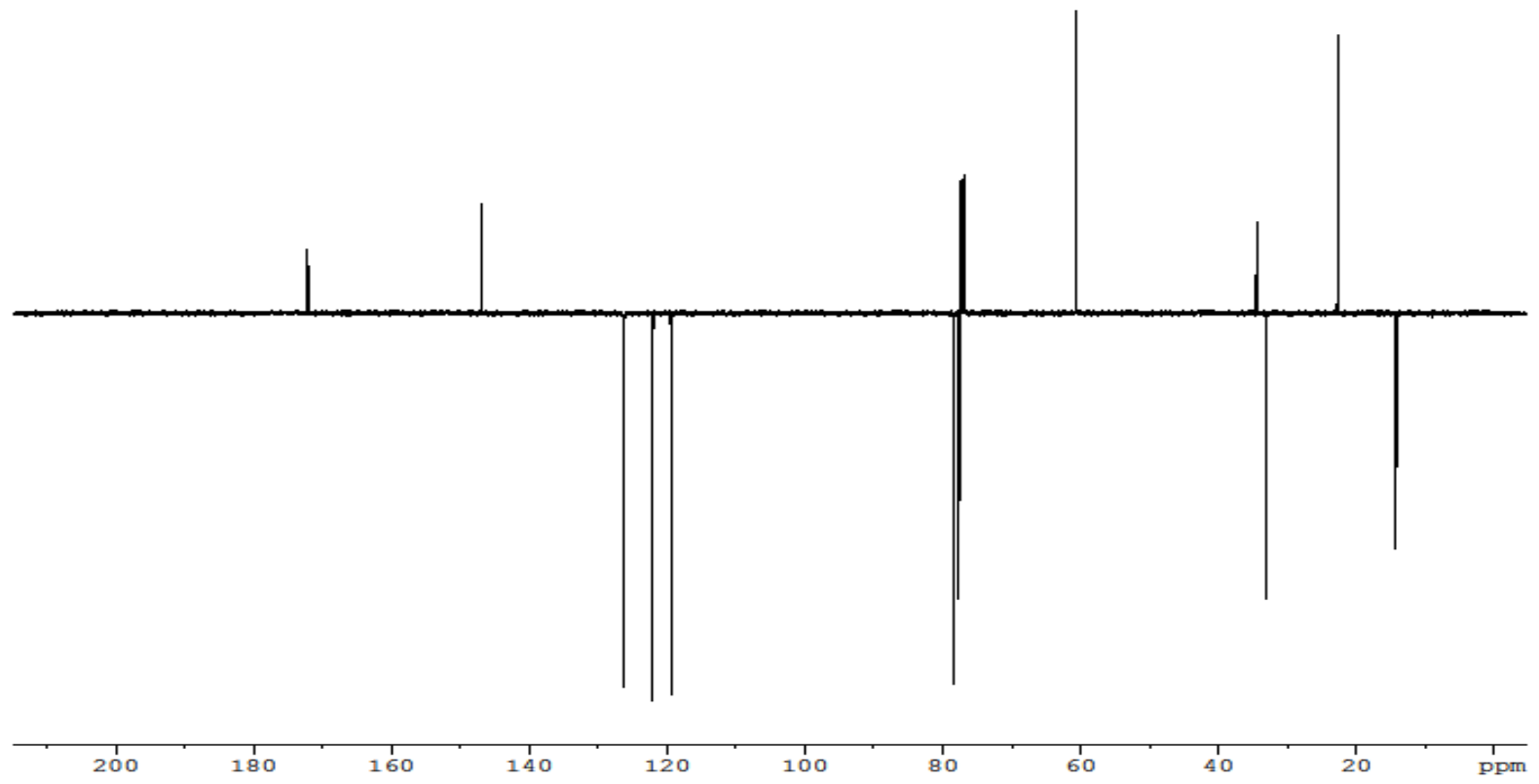

Figure S14. $100 \mathrm{MHz}^{13} \mathrm{C}$ NMR spectrum of cyclopropane $2 \mathrm{i}$ in $\mathrm{CDCl}_{3}$ 


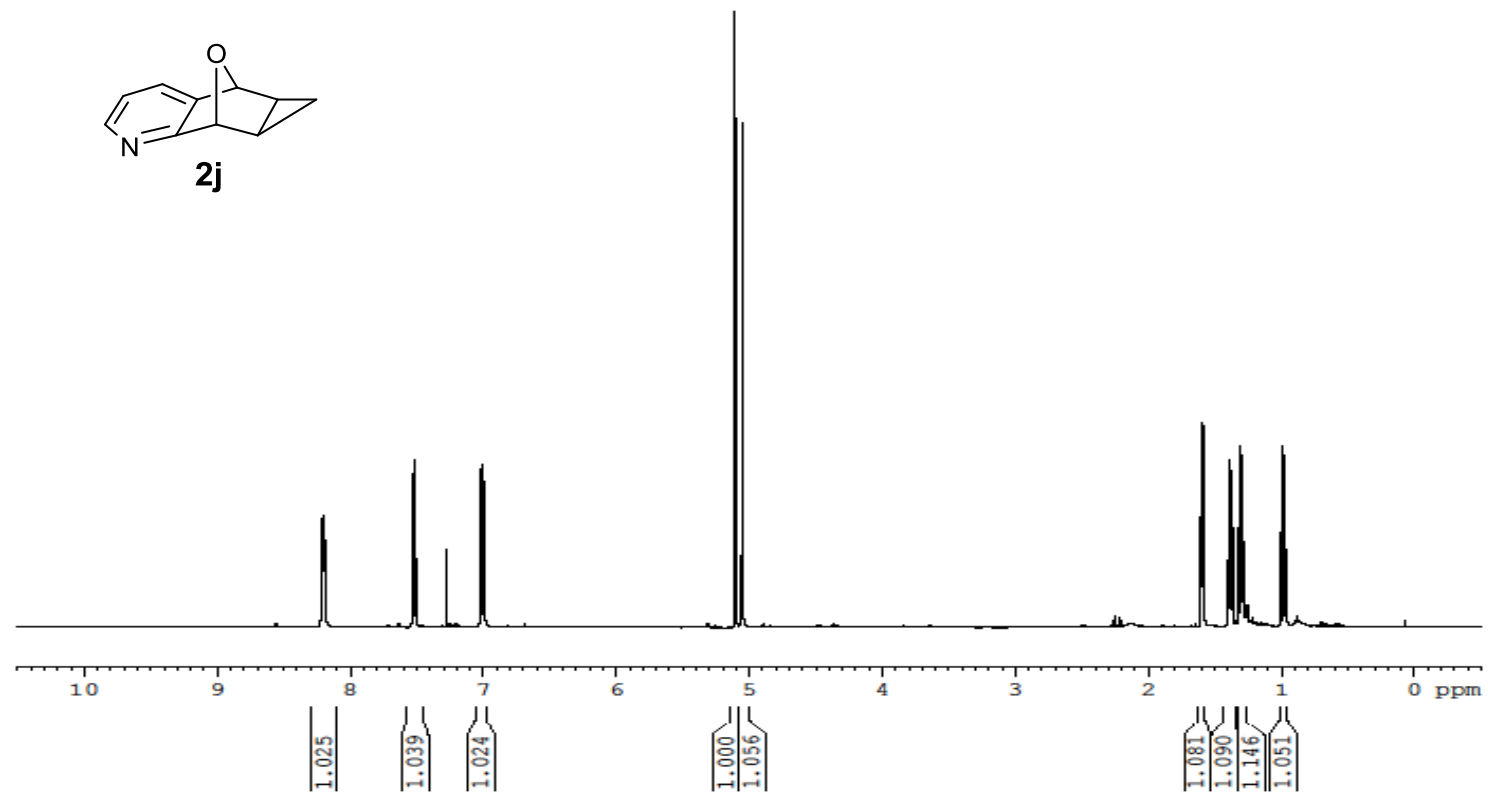

Figure S15. $400 \mathrm{MHz}{ }^{1} \mathrm{H}$ NMR spectrum of cyclopropane $2 \mathrm{j}$ in $\mathrm{CDCl}_{3}$

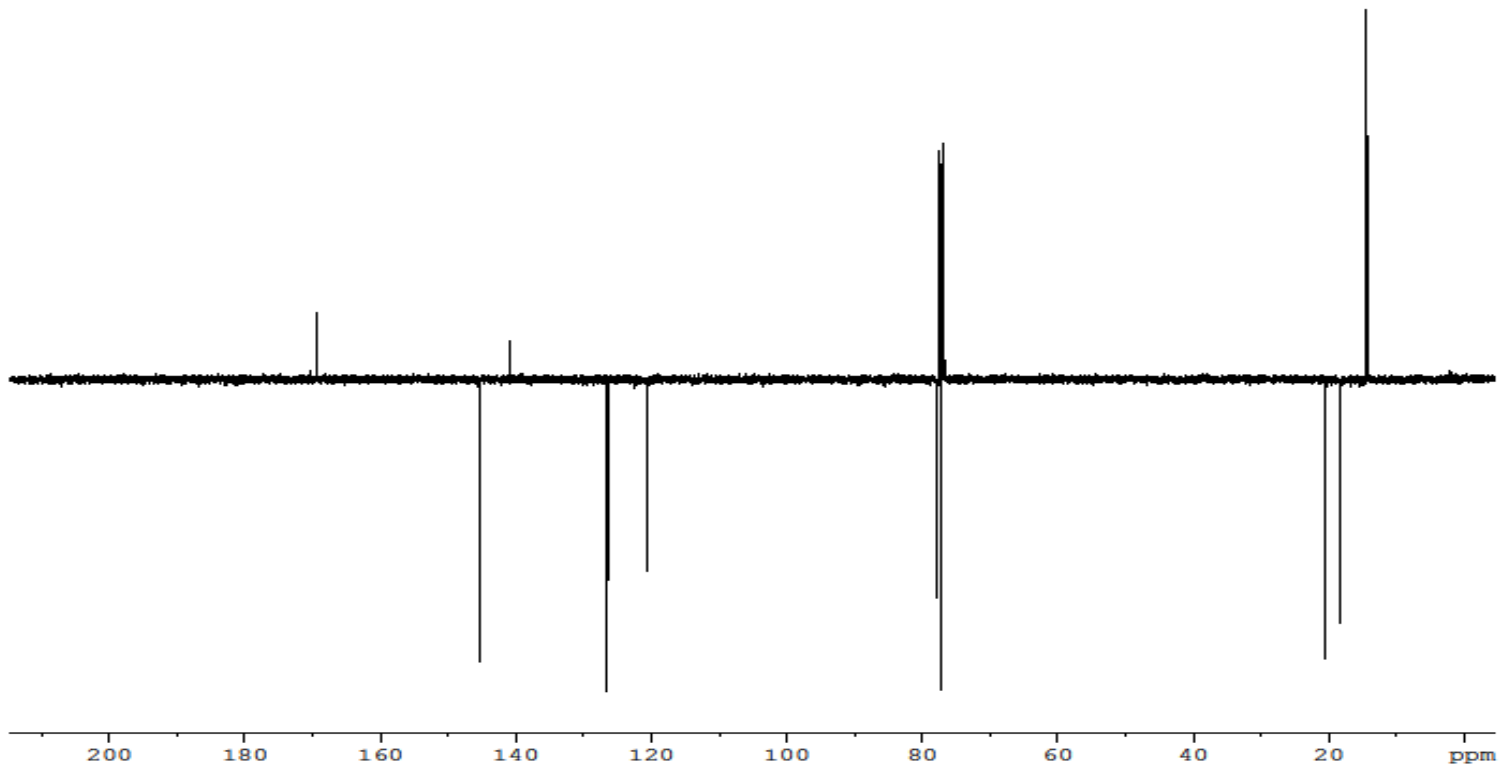

Figure S16. $100 \mathrm{MHz}^{13} \mathrm{C} \mathrm{NMR}$ spectrum of cyclopropane $2 \mathrm{j}$ in $\mathrm{CDCl}_{3}$ 


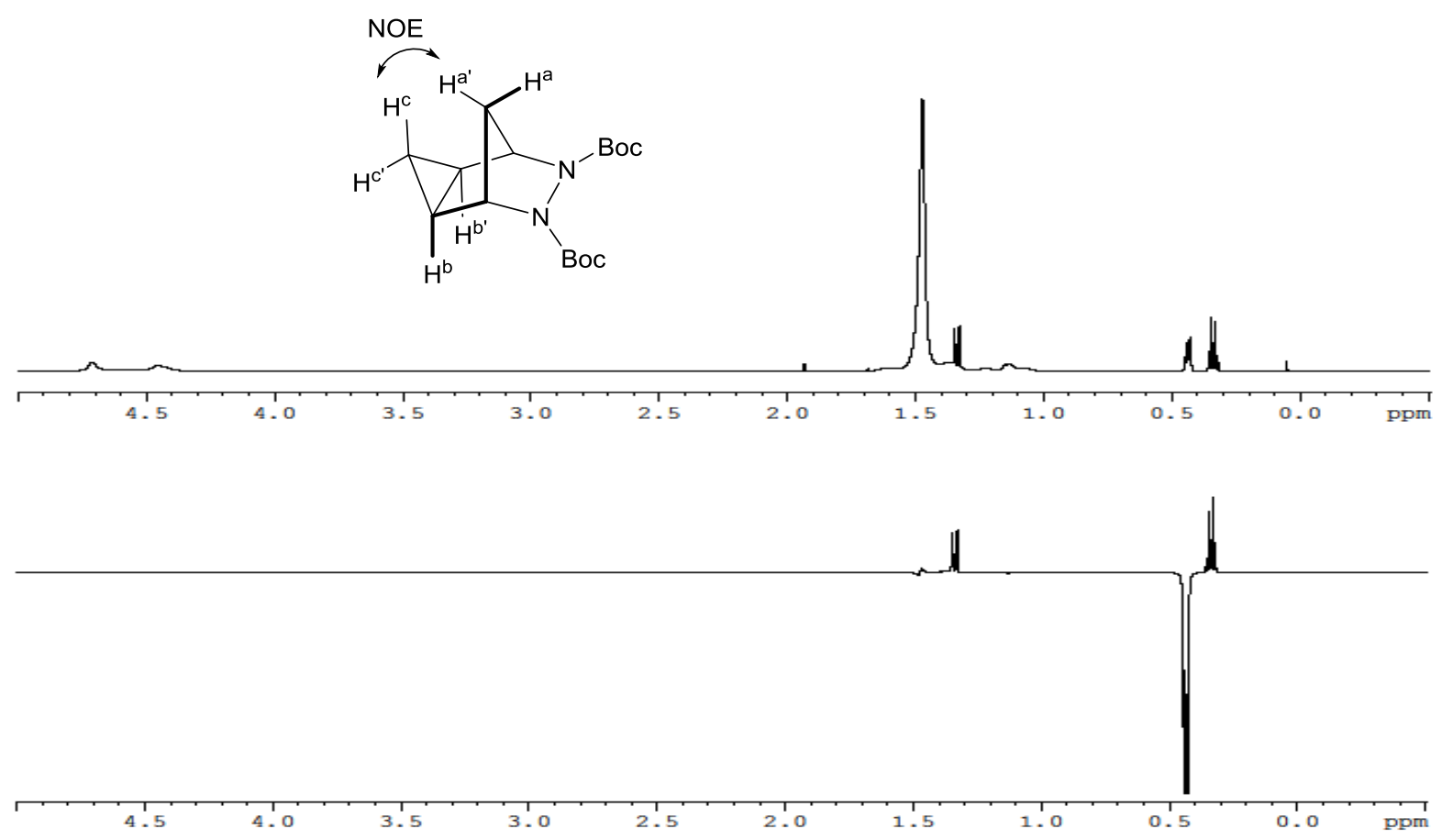

Figure S17. $600 \mathrm{MHz}$ selective gradient $\mathrm{NOE}$ spectrum of $6 \mathrm{a}$ in $\mathrm{CDCl}_{3}$

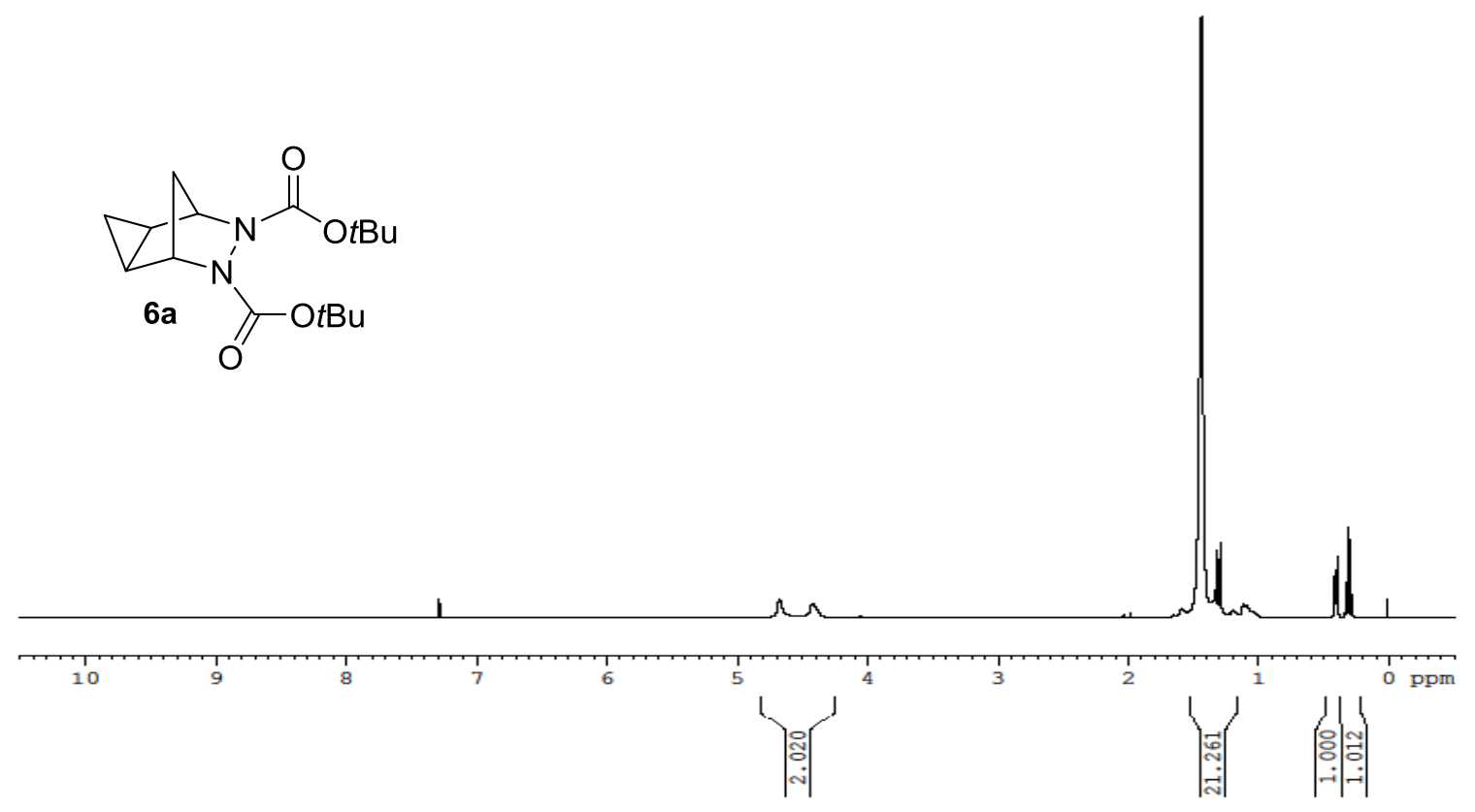

Figure S18. $400 \mathrm{MHz}^{1} \mathrm{H}$ NMR spectrum of cyclopropane $6 \mathrm{a}$ in $\mathrm{CDCl}_{3}$ 


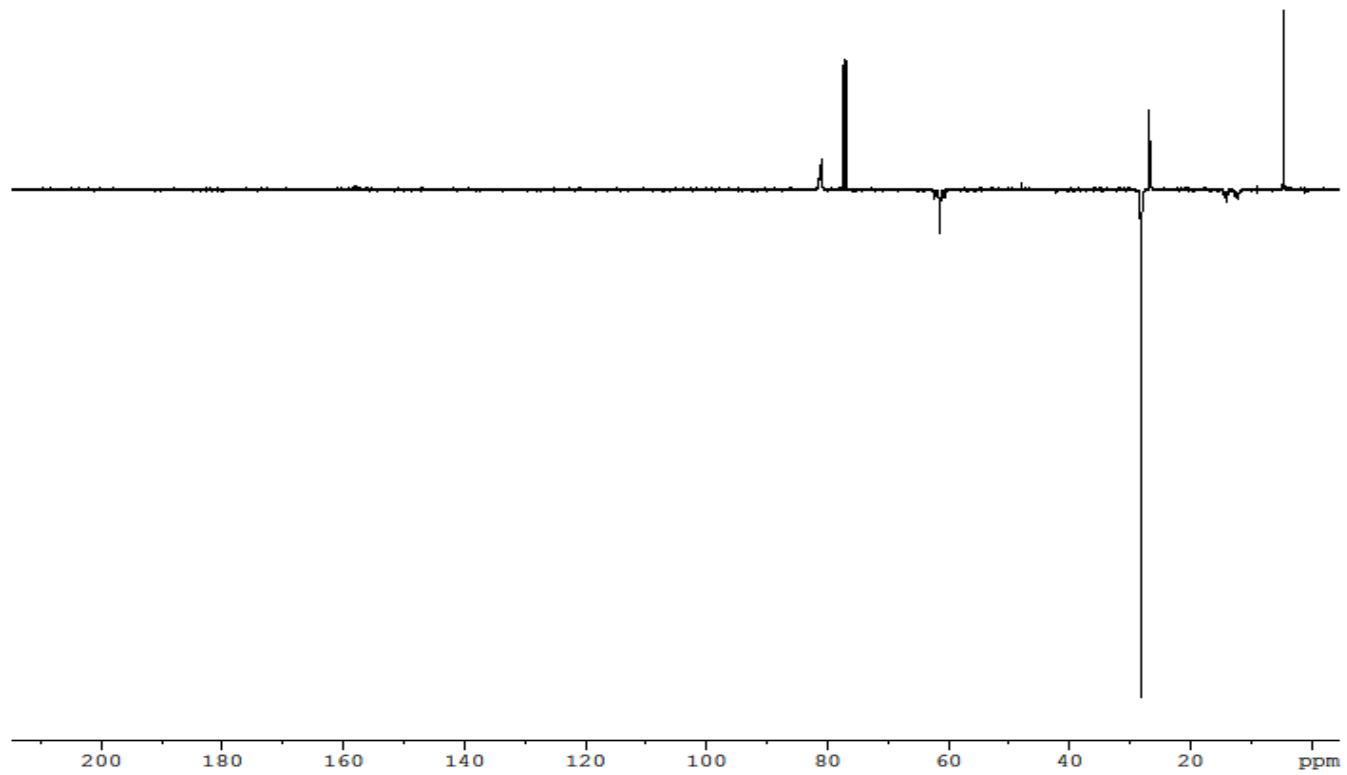

Figure S19. $125 \mathrm{MHz}^{13} \mathrm{C}$ NMR spectrum (JMOD) of cyclopropane $6 \mathrm{a}$ in $\mathrm{CDCl}_{3}$

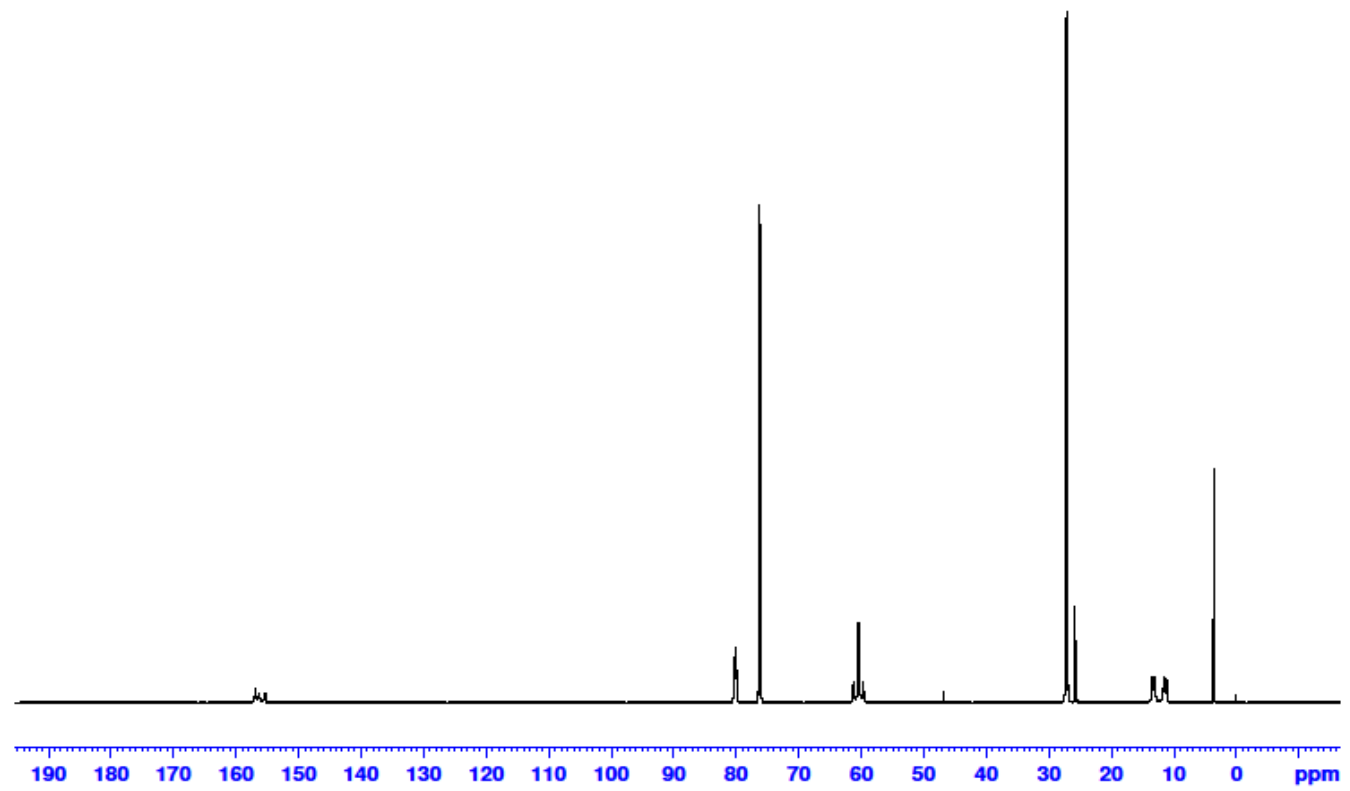

Figure $\mathrm{S20} .125 \mathrm{MHz}^{13} \mathrm{C}$ NMR spectrum of cyclopropane $6 \mathrm{a}$ in $\mathrm{CDCl}_{3}$ 


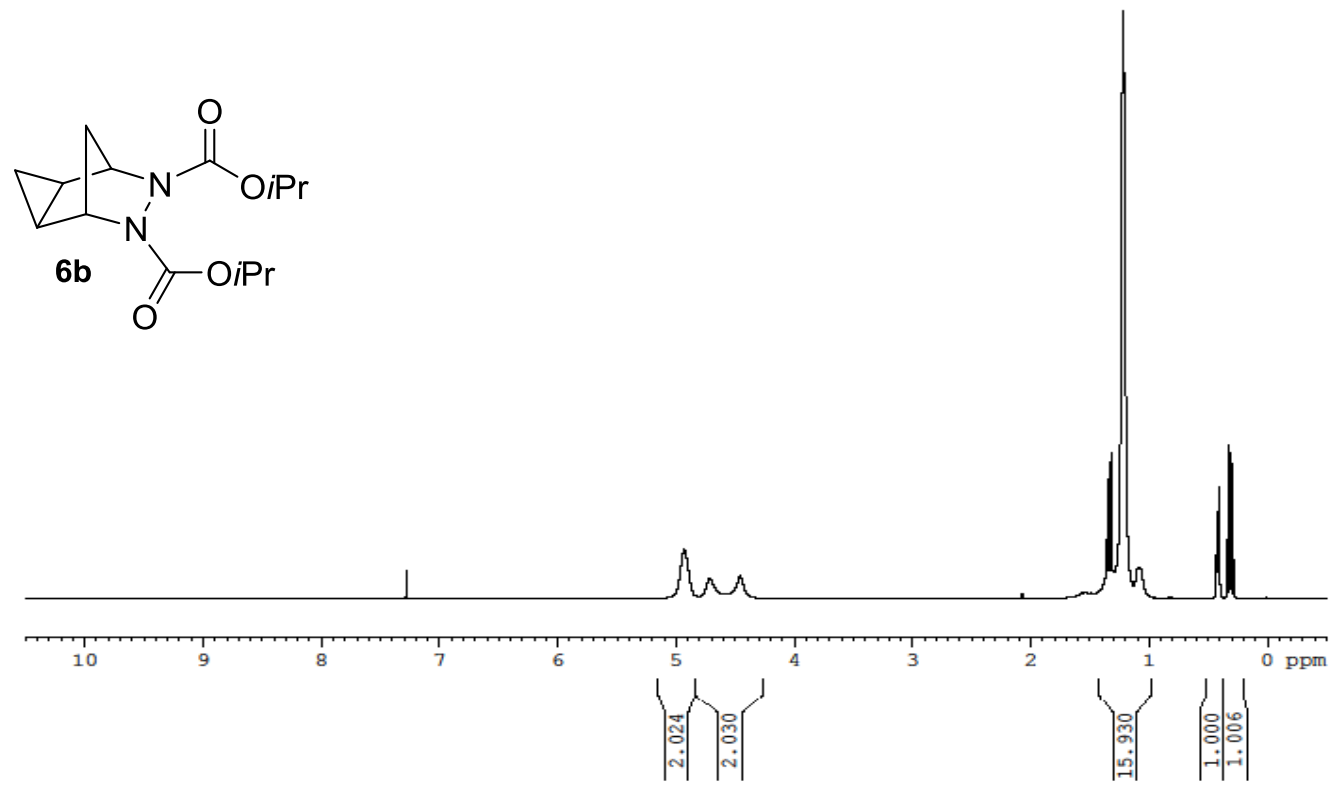

Figure S21. $400 \mathrm{MHz}^{1} \mathrm{H}$ NMR spectrum of cyclopropane $6 \mathrm{~b}$ in $\mathrm{CDCl}_{3}$

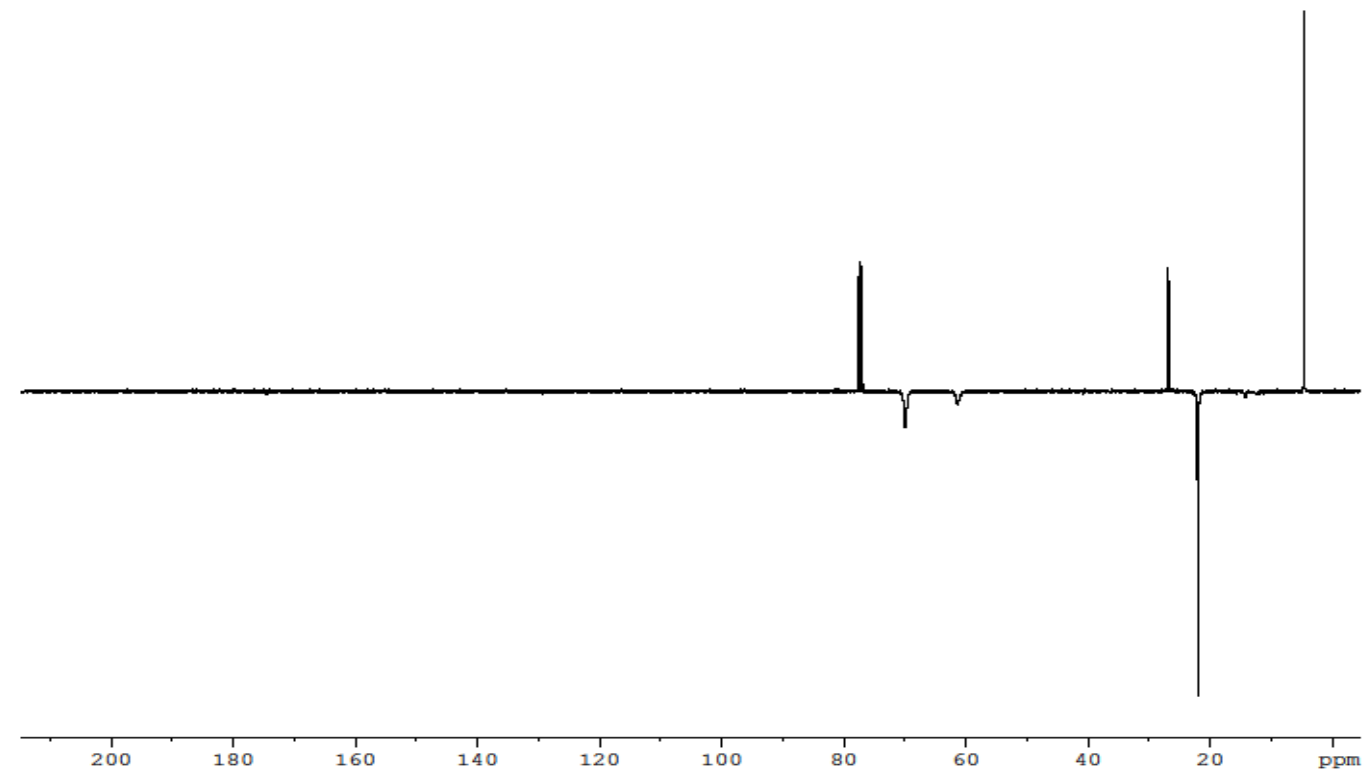

Figure S22. $100 \mathrm{MHz}{ }^{13} \mathrm{C}$ NMR spectrum (JMOD) of cyclopropane $6 \mathrm{~b}$ in $\mathrm{CDCl}_{3}$ 


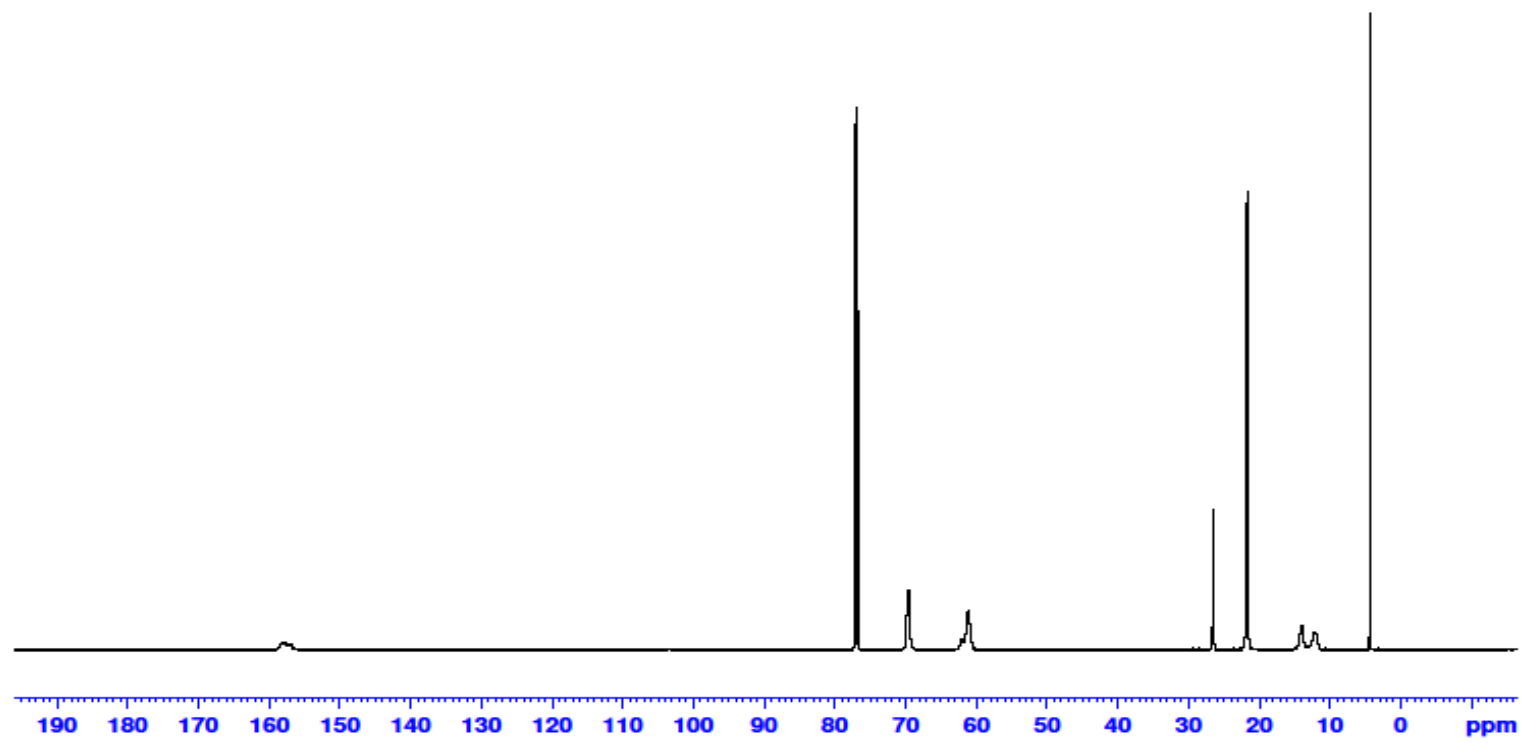

Figure S23. $125 \mathrm{MHz}^{13} \mathrm{C}$ NMR spectrum of cyclopropane $6 \mathrm{~b}$ in $\mathrm{CDCl}_{3}$

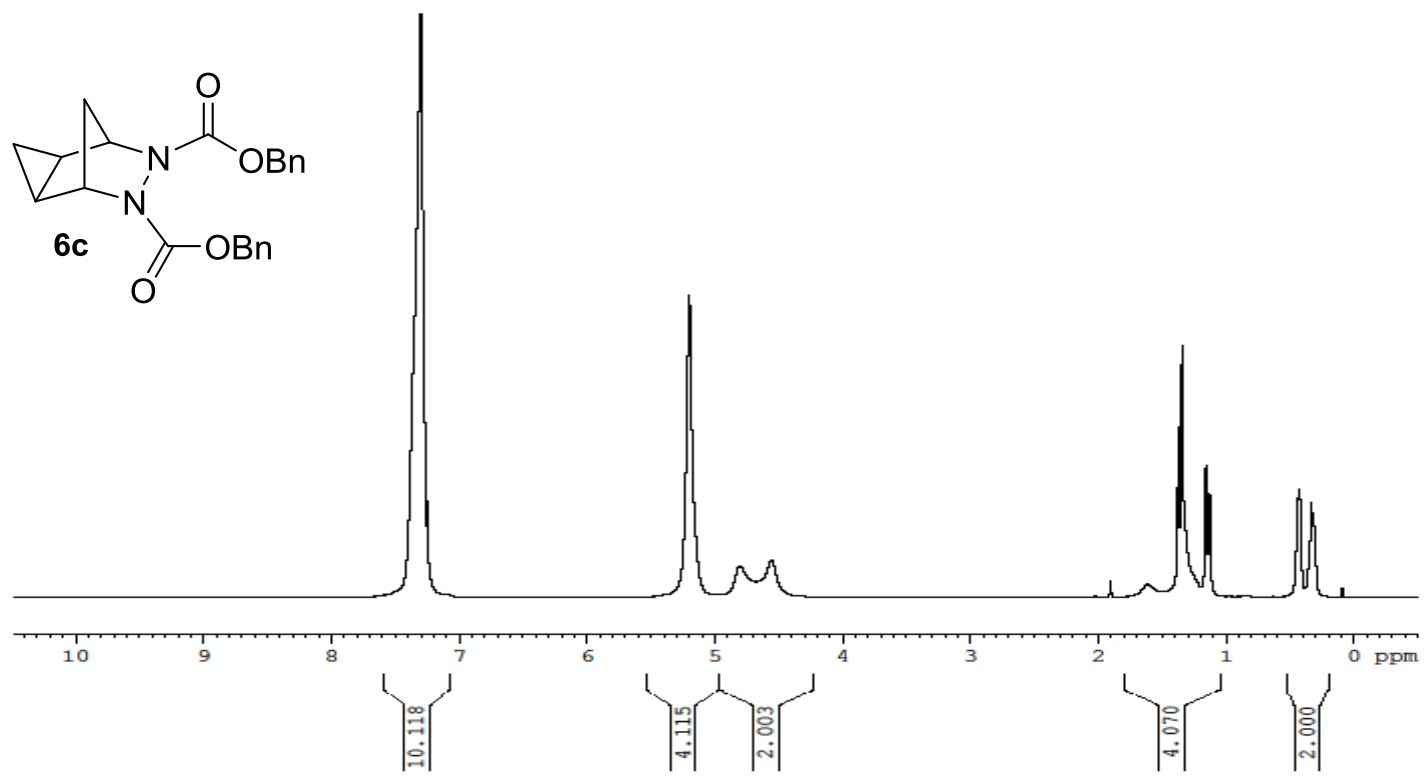

Figure S24. $400 \mathrm{MHz}^{1} \mathrm{H}$ NMR spectrum of cyclopropane $6 \mathrm{c}$ in $\mathrm{CDCl}_{3}$ 


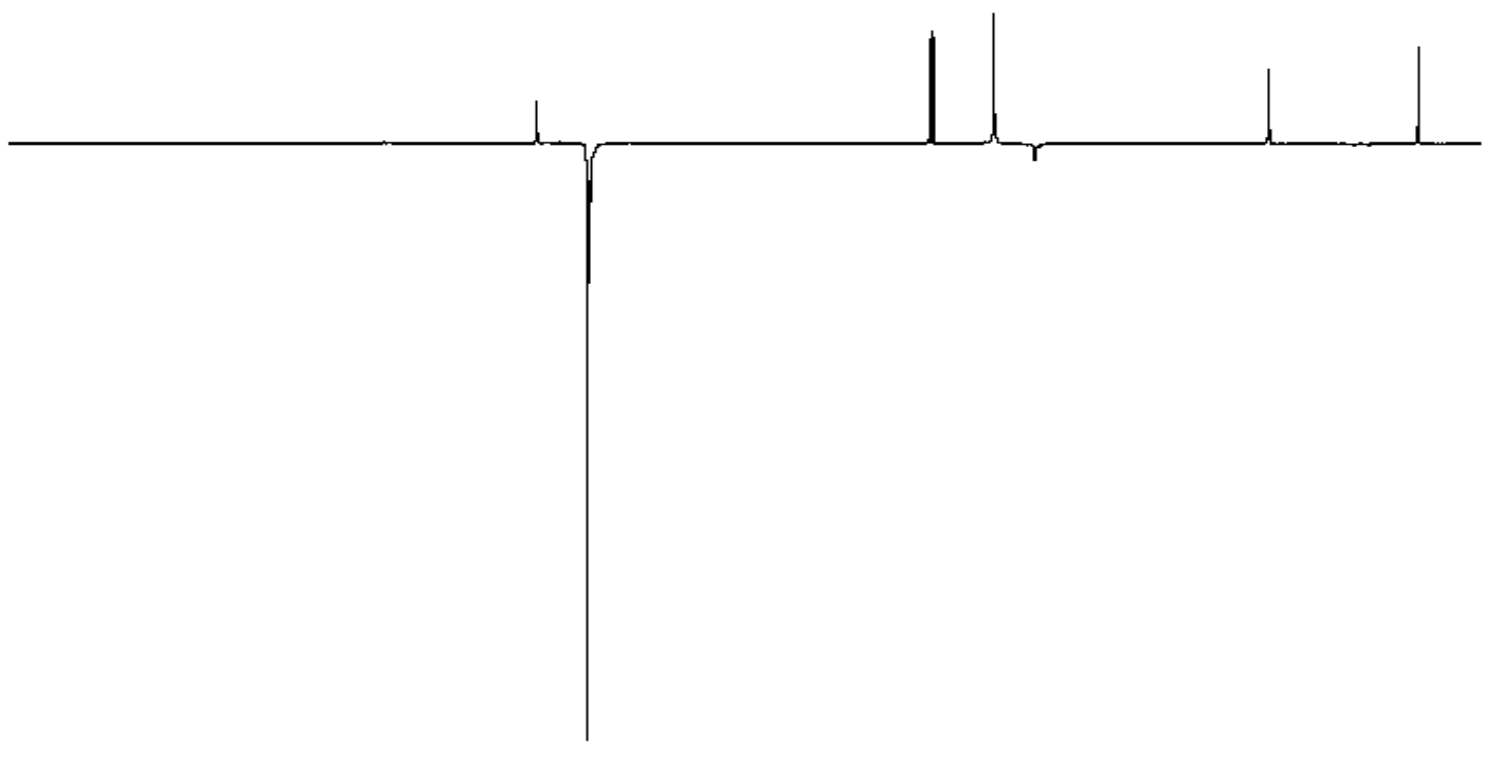

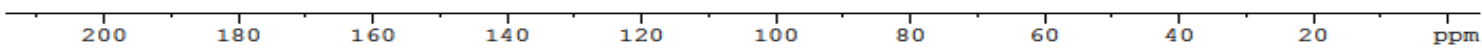

Figure S25. $100 \mathrm{MHz}{ }^{13} \mathrm{C}$ NMR spectrum (JMOD) of cyclopropane $6 \mathrm{c}$ in $\mathrm{CDCl}_{3}$

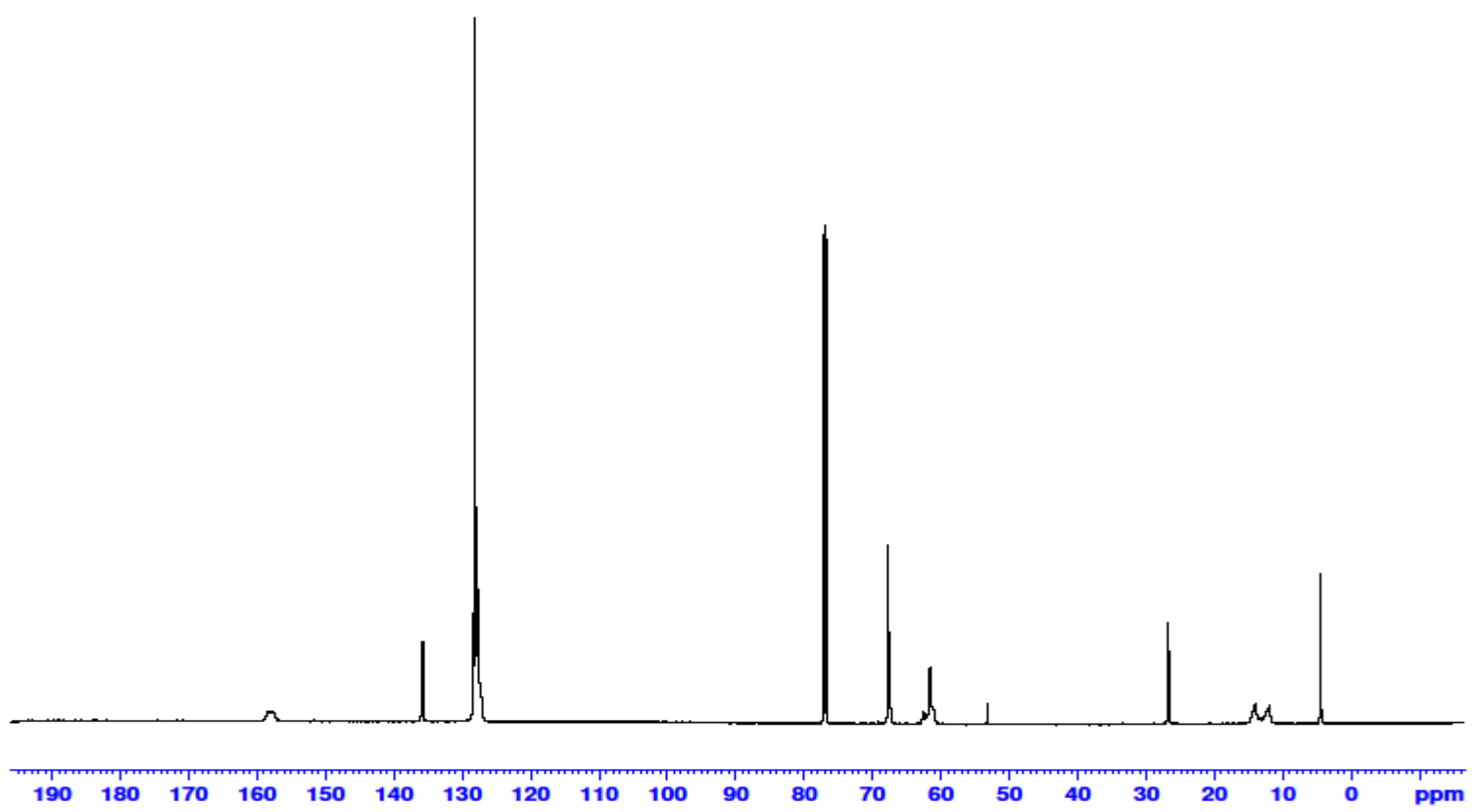

Figure S26. $125 \mathrm{MHz}^{13} \mathrm{C} \mathrm{NMR}$ spectrum of cyclopropane $6 \mathrm{c}$ in $\mathrm{CDCl}_{3}$ 